\title{
Fusion of the SRDX Motif to OsPIL11 or OsPIL16 Causes Rice Constitutively Photomorphogenic Phenotypes In Darkness
}

\section{Yaping Li}

Shandong Normal University

\section{Fang Zhang}

Institute of Genetics and Developmental Biology Chinese Academy of Sciences

Chongke Zheng

Shandong Academy of Agricultural Sciences

Jinjun Zhou

Shandong Academy of Agricultural Sciences

\section{Xiangxue Meng}

Shandong Normal University

\section{Shulin Niu}

Shandong Normal University

\section{Fan Chen}

Institute of Genetics and Developmental Biology CAS: Institute of Genetics and Developmental Biology

Chinese Academy of Sciences

Hui Zhang

Shandong Normal University

Xianzhi Xie ( $\nabla$ xzhxie2010@163.com)

Shandong Academy of Agricultural Sciences

\section{Research Article}

Keywords: rice, phytochrome interacting factor, constitutively photomorphogenic phenotypes, photosynthesis, auxin

Posted Date: September 13th, 2021

DOI: https://doi.org/10.21203/rs.3.rs-277064/v1

License: (c) (i) This work is licensed under a Creative Commons Attribution 4.0 International License.

Read Full License 
Version of Record: A version of this preprint was published at Plant Growth Regulation on November 12th, 2021. See the published version at https://doi.org/10.1007/s10725-021-00767-9. 


\section{Abstract}

Dark-grown seedlings develop skotomorphogenically. Because of the development of rice direct seeding cultivation systems, there is an increasing need for clarifying the molecular mechanism underlying rice skotomorphogenic development. It has been reported that SRDX motif, LDLDLELRLGFA, was able to convert a transcriptional activator into a strong repressor. In the present study, to explore the functions of PILs in rice skotomorphogenesis, we generated OSPIL 11-SRDX and OSPIL 16-SRDX transgenic lines by fusing the SRDX transcriptional repressor motif to the C-terminal of two members of the phytochrome interacting factor-like (OsPIL) family in rice (OsPIL11 and OsPIL16). The OsPIL 11-SRDX and OsPIL16$S R D X$ seedlings grown in darkness had constitutively photomorphogenic phenotypes with short coleoptiles and open leaf blades. The results of an RNA sequencing analysis revealed that the dark-grown OSPIL11-SRDX and OSPIL 16-SRDX lines had gene expression patterns similar to those of wild-type seedlings grown under red light. Kyoto Encyclopedia of Genes and Genomes pathway enrichment analyses indicated that the expression levels of genes related to photosynthesis, photosynthesisantenna proteins, and porphyrin and chlorophyll metabolism were up-regulated in the dark-grown OSPIL 11-SRDX and OSPIL 16-SRDX lines, whereas the expression of genes related to the auxin pathway was down-regulated. In contrast, the expression levels of these photosynthesis-related genes were downregulated in dark-grown transgenic seedlings overexpressing OSPIL 11 or OSPIL 16, which had exaggerated skotomorphogenesis. Considered together, our data indicate that OsPIL11 and OsPIL16 primarily function as transcriptional activators, at least in regards to promoting skotomorphogenesis and repressing the expression of photosynthesis-related genes.

\section{Introduction}

Skotomorphogenesis in the absence of light and photomorphogenesis in the presence of light are two contrasting plant developmental programs. Dark-grown Arabidopsis thaliana (Arabidopsis) plants have etiolated phenotypes with long hypocotyls, apical hooks, and closed and yellowish cotyledons (Leivar et al. 2008; Pham et al. 2018). In darkness, skotomorphogenic plant growth is necessary for photoautotroph survival. Regarding Arabidopsis, rapid and exaggerated hypocotyl elongation helps seedlings seek light. The tightly folded apical hook allows easy passage through the soil and protects the small cotyledons and underlying meristematic region from damage (Josse and Halliday 2008). Thus, clarifying the molecular mechanisms underlying skotomorphogenesis is an important research objective. There has recently been rapid progress in the characterization of Arabidopsis phytochrome interacting factors (PIFs) in terms of their functions mediating the switch from skotomorphogenesis to photomorphogenesis.

Phytochrome interacting factors, which form a subset of the basic helix-loop-helix (bHLH) transcription factor superfamily, are reportedly essential for maintaining the skotomorphogenic state of etiolated Arabidopsis seedlings (Castillon et al. 2007; Leivar and Monte 2014; Leivar and Quail 2011). The Arabidopsis PIF family comprises eight members (PIF1-8) (Lee and Choi 2017). Previous studies revealed that in darkness, a quadruple mutant (pifQ) lacking PIF1, PIF3, PIF4, and PIF5 resembles a wildtype (WT) seedling exposed to light, with short hypocotyls and expanded cotyledons (Kim et al. 2011; 
Leivar et al. 2009; Shin et al. 2009). Microarray analyses of the $p i f Q$ mutant and the subsequent Gene Ontology analysis indicated that PIF1, PIF3, PIF4, and PIF5 repress the expression of genes involved in chlorophyll biosynthesis and photosynthesis in plants grown in darkness (Shin et al. 2009; Zhang et al. 2013). Similarly, PIF1 and/or PIF3 suppress the expression of genes associated with chlorophyll biosynthesis and photosynthesis during de-etiolation (Chen et al. 2013; Liu et al. 2013).

Although the functions of Arabidopsis PIFs in skotomorphogenesis continue to be elucidated, the functions of rice PIF homologs affecting skotomorphogenesis remain unclear. Nakamura et al. (2007) identified six PIF-like (PIL) homologs in the rice genome (OSPIL 11-16). In darkness, the Arabidopsis pifQ mutant has constitutively photomorphogenic phenotypes. However, monogenic mutations at each of the four loci result in no or minimal visible effects on skotomorphogenesis. Various double and triple pif mutations result in increasingly photomorphogenic phenotypes in darkness (Kim et al. 2011; Leivar et al. 2008; Leivar et al. 2012; Lorrain et al. 2009; Shin et al. 2009; Stephenson et al. 2009). These results indicate that these four Arabidopsis PIFs functions in a highly redundant manner to control skotomorphogenic development.

It has been reported that ethylene-responsive element-binding factor (ERF)-associated amphiphilic repression (EAR) motif was able to convert a transcriptional activator into a strong repressor (Hiratsu et al. 2002; Ohta et al. 2001). Hiratsu et al maximized the potential repressive activity of the EAR motif repression domain, and the resultant repression domain, LDLDLELRLGFA, was designated as SRDX (Hiratsu et al. 2003). This technology was developed to study the consequences of silencing the target genes of individual transcription factors. Interestingly, SRDX dominant repressors may repress the transcription of their target genes as well as the expression of the genes targeted by other members of their respective gene families, thereby overcoming functional redundancy (Hiratsu et al. 2003; Mitsuda et al. 2007). In this study, to explore the functions of rice PILs in skotomorphogenesis, we fused the SRDXencoding DNA sequence to the $3^{\prime}$ end of OSPIL 11 and OSPIL 16. The OSPIL 11-SRDX and OSPIL 16-SRDX fusion constructs were expressed in WT Nipponbare plants. The resulting transgenic plants had constitutively photomorphogenic phenotypes in the absence of light. Molecular analyses revealed that genes related to photosynthesis and the auxin pathway were enriched among the differentially expressed genes (DEGs). Our findings suggest that either OsPIL11 or OsPIL16 is necessary for maintaining rice skotomorphogenesis.

\section{Materials And Methods}

\subsection{Plant materials}

This study was completed with japonica rice varieties, including Nipponbare (NIP), Wuyunjing7 (WYJ7), Yanfeng47 (YF47), Shengdao14 (SD14), Jingdao818 (JD818), Jinyuan45 (JY45), Ningjing44 (NJ), Songjing378 (SJ378), Yanjing44 (YJ44) and Nanjing46 (NJ46), as well as indica rice varieties, including 9311, Huanghuazhan (HHZ), Peiai64 (PA64), Minghui63 (MH63), Shuhui498 (SH498), Pokkali, Dular, Yixiang1B (YX1B), Haidao86 (HD86), and Jingang30 (JG30). 


\subsection{Phenotypes of dark-grown seedlings}

Rice seeds were surface-sterilized and then sown on $0.4 \%(\mathrm{w} / \mathrm{v})$ agar. After an overnight incubation at 4 ${ }^{\circ} \mathrm{C}$, the seeds were germinated at $28 \pm 1{ }^{\circ} \mathrm{C}$ and grown in darkness for 9 days. The seedlings were then photographed.

\subsection{Generation of transgenic lines}

The previously described OsPIL 11-overexpression (OsPIL11-OX) vector (Li et al. 2012b) and OsPIL16overexpression (OsPIL 16-OX) vector (He et al. 2016) were used for generating transgenic lines. In these two vectors, OSPIL 11 and OSPIL 16 were subcloned between the maize ubiquitin gene promoter and the nos terminator. To obtain the SRDX sequence, two single-stranded DNA fragments (5'PGATCCATGGCACTGGATCTGGATCTGGAACTGCGCCTGGGCTTTGCGTAAG $\underline{O H}^{-3}$ and 5'PAATTCTTACGCAAAGCCCAGGCGCAGTTCCAGATCCAGATCCAGTGCCATG ${ }_{O H}{ }^{-3}$ ') were synthesized by Sangon Biotech Co., Ltd. (Shanghai, China). After denaturing at $98{ }^{\circ} \mathrm{C}$ for $3 \mathrm{~min}$, the sequences were annealed at $55^{\circ} \mathrm{C}$ for $5 \mathrm{~min}$ to produce double-stranded $S R D X$, which encodes a peptide containing the SRDX domain (LDLDLELRLGFA) (Hiratsu et al. 2003). The ends of the SRDX sequence included BamH I (PGATCC-, underlined) and ECOR I (-G- and -AATTC-, underlined) sites for the subcloning of the doublestranded SRDX sequence into the pBluescript II SK (+) vector to generate the pBS-SRDX vector.

To produce the OSPIL11-SRDX and OSPIL16-SRDX fusion constructs, the OSPIL 11 and OSPIL 16 open reading frames without the stop codon were amplified by PCR using the OsPIL11-OX and OsPIL 16-OX vectors as templates. The primer pair used for amplifying OsPIL 11 was OsPIL11 Xbal F1 (5'AATCTAGATGAACCAGTTCGTCCCTG-3', Xba I site underlined) and PIL11 BamHI R1 (5'ATGGATCCGGAGTCAGCGGCTGC-3', BamH I site underlined). The primer pair used for amplifying OsPIL 16 was OsPIL16 Xbal F1 (5'-AATCTAGAATGCTACGCGGGAACGAC-3', Xba I site underlined) and PIL16 BamHI R1 (5'-AAGGATCCCGCCTGCTTCACGGCGGGG-3', BamH I site underlined). The PCR products were digested with $\mathrm{Xba} \mathrm{I/BamH} \mathrm{I} \mathrm{and} \mathrm{inserted} \mathrm{into} \mathrm{the} \mathrm{pBS-SRDX} \mathrm{vector,} \mathrm{resulting} \mathrm{in} \mathrm{pBS}$ OsPIL11-SRDX and pBS-OsPIL16-SRDX vectors. To construct the OsPIL11-SRDX and OsPIL16-SRDX plant expression vectors, the OSPIL 11-SRDX and OSPIL 16-SRDX sequences were separately amplified by PCR using either pBS-OsPIL11-SRDX or pBS-OsPIL16-SRDX as the template. The primer pair used for amplifying OsPIL 11-SRDX was OsPIL11 Sfil F1 (5'-

AGGCCAAATCGGCCATGAACCAGTTCGTCCCTGATTGG-3', Sfi I site underlined) and SRDX Sfil R1 (5'TGGCCCTTATGGCCTTACGCAAAGCCCAGGCGCAGTTC-3', Sfi I site underlined). The primer pair used for amplifying OsPIL 16-SRDX was OsPIL16 Sfil F1 (5'-AGGCCAAATCGGCCATGCTACGCGGGAACGACACCG-3', Sfi I site underlined) and SRDX Sfil R1. The PCR products were digested with $S f i$ I and inserted into the pRiceFOX vector, resulting in the pRiceFOX-OsPIL11-SRDX and pRiceFOX-OsPIL16-SRDX plant expression vectors.

The plant expression vectors were transformed into Agrobacterium tumefaciens strain EHA105 by electroporation and the resulting bacteria cultures were used for the transformation of rice (Oryza sativa 
cv Nipponbare).

\subsection{DNA extraction and southern blot analysis}

Genomic DNA was isolated and purified from the OSPIL 16-SRDX lines, OSPIL11-SRDX lines, and WT plants at the three-leaf stage following Murray and Thompson (1980). DNA was digested with Hind III and transferred to a nylon membrane (Hybond-N+; Amersham, Buckinghamshire, UK) after electrophoresis on a $0.8 \%$ agarose gel. Hpt // gene was selected as the marker gene. The detailed protocol was the same as described by $\mathrm{He}$ et al. (2016).

\subsection{Subcellular localization of OsPIL16-SRDX-GFP and OsPIL11-SRDX-GFP}

To construct the OsPIL11-SRDX-GFP and OsPIL16-SRDX-GFP fusion proteins, the OsPIL 11-SRDX and OsPIL 16-SRDX sequences were separately amplified by PCR using either pBS-OsPIL11-SRDX or pBSOsPIL16-SRDX as the template. The primer pair used for amplifying OSPIL 11-SRDX was OsPIL11 F2 (5'gagaacacgggggactctagaATGAACCAGTTCGTCCCTGATT-3', 21 bases homologous to the ends of the linearized vector in lowercase letters) and SRDX R2 (5'-

tcatgcctccgcggeggatccaCGCAAAGCCCAGGCGCAG-3', 22 bases homologous to the ends of the linearized vector in lowercase letters). The primer pair used for amplifying OsPIL 16-SRDX was OsPIL16 F2 (5'gagaacacgggggactctagaATGCTACGCGGGAACGACA-3', 21 bases homologous to the ends of the linearized vector in lowercase letters) and SRDX R2. The PCR products were subcloned between the cauliflower mosaic virus 35S promoter and the GFP start codon in the pBI221-GFP vector according to the In-Fusion ${ }^{\circledR}$ HD Cloning Kit User Manual (Clontech, CA, USA). The resulting plasmid DNA was transiently introduced into rice protoplasts isolated from 10-day-old etiolated NIP seedlings using the polyethylene glycol method. The OsGEN1-mCherry protein was used as a nuclear marker. After an overnight incubation in darkness, fluorescence was observed using the FluoView FV1000 fluorescence confocal microscope (Olympus, Tokyo, Japan).

\subsection{Measurement of coleoptile and mesocotyl lengths}

Surface-sterilized seeds of the NIP, OsPIL 11-SRDX, OSPIL 16-SRDX, OSPIL 11-OX, and OsPIL 16-OX lines were sown on $0.4 \%(\mathrm{w} / \mathrm{v})$ agar and then incubated in darkness at $28{ }^{\circ} \mathrm{C}$ for 9 days. The seedlings were then photographed, after which the coleoptile and mesocotyl lengths were measured using a ruler.

\subsection{Transcriptome sequencing}

Surface-sterilized seeds of the WT control and the OSPIL 11-SRDX (\#2 and \#3) and OSPIL 16-SRDX (\#8 and \#9) transgenic lines were sown on $0.4 \%(\mathrm{w} / \mathrm{v})$ agar and incubated overnight at $4{ }^{\circ} \mathrm{C}$. The seeds were then incubated in darkness (D) or under red light (R) at $28{ }^{\circ} \mathrm{C}$ for 6 days. The above-ground plant parts were harvested for transcriptome sequencing analyses, which were completed by Novogene Biotech (Tianjin, China) using standard Illumina protocols. The clean reads were aligned to the assembled transcriptome using the Bowtie2 program (version 2.3.4.1) (Langmead and Salzberg 2012). Following the alignment, raw read counts for each transcript were determined using RSEM (version 1.2.31) (Li and Dewey 2011) 
and then normalized to FPKM (fragments per kilobase exon per million mapped fragments). Raw counts were analyzed using the DESeq2 package (Love et al. 2014) to identify DEGs, which were defined as genes with a false discovery rate adjusted $P<0.05$ and an expression level fold-change $\geq 2$. A Kyoto Encyclopedia of Genes and Genomes (KEGG) analysis was performed using the clusterProfiler R package (version 3.4.4) (Yu et al. 2012) to identify the significantly enriched pathways among the DEGs.

\subsection{Quantitative real-time ( $q R T)-P C R$ analysis}

Total RNA was isolated from the seedlings grown in darkness for 6 days using RNAiso Plus (TaKaRa, Dalian, China). First-strand cDNA was synthesized using the PrimeScript ${ }^{\mathrm{TM}} \mathrm{RT}$ reagent Kit with gDNA Eraser (Perfect Real Time) (TaKaRa). The qRT-PCR analysis was performed using the ABI Q5 PCR System (Applied Biosystems, Foster City, CA, USA) and TB Green® Premix Ex Taq ${ }^{\mathrm{TM}}$ II (Tli RNaseH Plus) (TaKaRa). The expression level of each target gene was derived from its signals normalized against either $e E F-1 a$ (AK061464) or ACTIN (LOC4333919) using the $\triangle C$ CT method (Jain et al. 2006; Livak and Schmittgen 2001). The qRT-PCR analysis was completed using three biological replicates. Primer details are listed in Table S1.

\subsection{Light sources and intensities}

The R sources comprised LED lighting units (IS-big ${ }^{\circledR}$, ISL-305 X 302-RRRR; CCS Inc., Kyoto, Japan; light emission surface: $300 \times 300 \mathrm{~mm}$; 1,512 LEDs were equally arranged on a panel) with power supply units (ISC-101-4; CCS Inc.). Plants were irradiated with LED light (15 mmol m $\mathrm{m}^{-2} \mathrm{~s}^{-1}$ for $\mathrm{R}$ ) in a plant growth chamber (LH-55LED-SS; Nippon Medical \& Chemical Instruments Co., Ltd., Osaka, Japan).

\subsection{Data analysis of coleoptile length}

All results are expressed as mean values \pm standard error (SE) based on more than 20 seedlings. Statistical significance was assessed using Student's $t$-test. Probability values of less than 0.05 were considered to be statistically significant. A single asterisk $\left({ }^{*}\right)$ and double asterisks $\left({ }^{*}\right)$ represent significance at the levels of 0.05 and 0.01 , respectively.

\section{Results}

\subsection{Characteristics of rice skotomorphogenic phenotypes}

Dark-grown Arabidopsis seedlings have etiolated phenotypes with long hypocotyls, apical hooks, and closed and yellowish cotyledons (Leivar et al. 2008). In this study, we examined the phenotypes of darkgrown japonica and indica rice seedlings. The japonica rice varieties grown in darkness had long coleoptiles. In 9-day-old seedlings grown in darkness, yellowish and rolled leaves were either wrapped by coleoptiles or grew out of coleoptiles (Fig. 1a, upper panel). Although the morphological characteristics of the indica seedlings were similar to those of japonica seedlings, some indica varieties grown in darkness had longer mesocotyls (Fig. 1b, upper panel). However, seedlings grown under R conditions had shorter 
coleoptiles, green leaves, and expanded leaf blades (Fig 1a and b, lower panels). These results indicate that relatively long coleoptiles and mesocotyls as well as yellowish and rolled leaf blades are typical characteristics of dark-grown rice seedlings.

\subsection{OsPIL16-SRDX and OsPIL11-SRDX lines grown in darkness had constitutively photomorphogenic phenotypes}

In Arabidopsis, PIFs negatively regulate light responses by repressing photomorphogenesis and maintaining the skotomorphogenic state of etiolated seedlings in darkness (Leivar and Monte 2014; Leivar et al. 2008). To clarify the roles of rice PIFs in maintaining etiolated seedling development, we produced transgenic lines expressing the OSPIL 16-SRDX fusion construct under the control of the ubiquitin gene promoter (Fig. 2a). Three independent $\mathrm{T}_{3}$ lines (\#4, \#8, and \#9) were used to analyze the

role of OsPIL16-SRDX based on the result of Southern blot analysis and their high expression levels (Fig. $2 \mathrm{~b}$ and $\mathrm{c}$ ). A comparison of the WT and OSPIL 16-SRDX lines grown in darkness for 9 days revealed that the coleoptiles of the OSPIL 16-SRDX lines $(0.58 \pm 0.01 \mathrm{~cm}, 0.50 \pm 0.01 \mathrm{~cm}$, and $0.44 \pm 0.01 \mathrm{~cm}$ in lines \#4, $\# 8$, and \#9, respectively) were shorter than the WT coleoptile $(3.76 \pm 0.02 \mathrm{~cm})$ (Fig. $2 \mathrm{~d}$ and e). Moreover, the second leaf blades of the OSPIL 16-SRDX lines were expanded, in contrast to the rolled leaf blades of the WT seedlings (Fig. 2d). Therefore, OsPIL 16-SRDX lines had photomorphogenic phenotypes (i.e., short coleoptiles and expanded leaf blades) similar to the WT seedlings grown under R (Fig. S1). These results imply that OsPIL16-SRDX negatively regulates rice skotomorphogenesis.

An earlier phylogenetic analysis revealed that among the six family members, the greatest genetic diversity was between OSPIL 11 and OSPIL 16 (Nakamura et al. 2007). To further explore whether other PIF members are also involved in rice skotomorphogenesis, we added the SRDX domain to the C-terminal of OsPIL11 (Fig. 2a). Two independent $\mathrm{T}_{4}$ OsPIL 11-SRDX transgenic lines (\#2 and \#3) were used to analyze the role of OsPIL11-SRDX based on the result of Southern blot analysis and their high expression levels (Fig. $2 \mathrm{~b}$ and c). Similar to the OSPIL 16-SRDX lines, the OSPIL11-SRDX lines had shorter coleoptiles (0.87 \pm $0.03 \mathrm{~cm}$ and $1.72 \pm 0.01 \mathrm{~cm}$ in lines \#2 and \#3, respectively) than the WT seedlings $(3.76 \pm 0.02 \mathrm{~cm}$ ) in darkness (Fig. $2 \mathrm{~d}$ and e). These observations suggest that OsPIL11-SRDX negatively regulates rice skotomorphogenesis.

\subsection{Subcellular localization of OsPIL11-SRDX and OsPIL16-SRDX proteins}

To examine the subcellular localization of OsPIL11-SRDX and OsPIL16-SRDX, we generated constructs for the expression of GFP-tagged fusion proteins under the control of the cauliflower mosaic virus $35 \mathrm{~S}$ promoter. The constructs were transiently expressed in rice protoplasts. The OsPIL11-SRDX-GFP and OsPIL16-SRDX-GFP signals were detected in the nucleus (Fig. 3). In the control protoplasts expressing GFP alone, fluorescent signals were detected in the cytoplasm and nucleus (Fig. 3). These results indicate that OsPIL11-SRDX and OsPIL16-SRDX are localized in the nucleus.

\subsection{The OsPIL16-SRDX and OsPIL11-SRDX seedlings grown in darkness have gene expression profiles similar to those of wild-type seedlings grown under red light}


To investigate the role of PIFs in maintaining rice skotomorphogenesis at the genome level, we performed an RNA sequencing (RNA-seq) analysis of dark-grown OsPIL 16-SRDX (\#8 and \#9), R-grown WT, and darkgrown WT seedlings. The data revealed 8,020 DEGs between the dark-grown OsPIL16-SRDX [OsPIL16SRDX(D)] and the dark-grown WT [WT(D)] (Table S2). Of these DEGs, the expression levels of 4,129 and 3,891 genes were up-regulated and down-regulated, respectively, in the OsPIL 16-SRDX lines (Fig. S2, Table S2), implying that OSPIL16-SRDX positively and negatively regulates gene expression. Furthermore, 10,526 DEGs were detected between WT(D) seedlings and WT(R) seedlings (i.e., WT seedlings exposed to R) (Table S3). A comparison of the two DEG sets indicated that approximately $49.8 \%$ of the DEGs between OsPIL16-SRDX(D) and WT(D) were also differentially expressed between WT(R) and WT(D) (Fig. 4a). These results indicate that dark-grown OSPIL16-SRDX seedlings have expression patterns that are similar to those of the R-grown WT control.

Because OsPIL11-SRDX lines had phenotypes that resembled those of OSPIL16-SRDX lines in darkness (Fig. 2), we also performed an RNA-seq analysis of OsPIL 11-SRDX lines. We detected 7,268 DEGs between the dark-grown OsPIL 11-SRDX [OsPIL11-SRDX(D)] and the WT(D) seedlings (Table S4). A comparison of the DEGs between OsPIL11-SRDX(D) and WT(D) and the DEGs between WT(R) and WT(D) revealed that approximately $52.0 \%$ of the DEGs between OsPIL11-SRDX(D) and WT(D) were also differentially expressed between WT(R) and WT(D) (Fig. 4a). These results indicate that the dark-grown OsPIL 11-SRDX and R -grown WT seedlings have similar gene expression patterns.

We also compared the DEGs between OsPIL16-SRDX(D) and WT(D) with the DEGs between OsPIL11SRDX(D) and WT(D). Approximately $73.6 \%(5,903$ of 8,020$)$ of the DEGs between OsPIL16-SRDX(D) and WT(D) were also differentially expressed between OsPIL11-SRDX(D) and WT(D), whereas about $81.2 \%$ $(5,903$ of 7,268$)$ of the DEGs between OsPIL11-SRDX(D) and WT(D) were also differentially expressed between OsPIL16-SRDX(D) and WT(D) (Fig. 4a). Accordingly, OsPIL 11-SRDX and OsPIL 16-SRDX seedlings grown in darkness have highly similar gene expression profiles.

\subsection{OsPIL11-SRDX and OsPIL16-SRDX promote the expression of photosynthesis-related genes in darkness, but overexpression of OsPIL11 and OsPIL16 has the opposite effect.}

Because the photomorphogenic phenotypes of the OSPIL11-SRDX and OSPIL 16-SRDX lines were similar to those of R-grown WT seedlings, we speculated that the shared DEGs among OsPIL11-SRDX(D)/WT(D), OsPIL16-SRDX(D)/WT(D), and WT(R)/WT(D) affect rice skotomorphogenesis. We detected 3,183 shared DEGs among the three DEG sets (Fig. 4a, Table S5). Of these DEGs, the expression levels of 1,239 and 1,320 genes were respectively up-regulated and down-regulated in the OsPIL11-SRDX(D), OsPIL16SRDX(D), and WT(R) seedlings (Fig. S2, Tables S6 and S7). The enriched KEGG pathways among the shared DEGs were associated with photosynthesis. More specifically, photosynthesis, photosynthesisantenna proteins, and porphyrin and chlorophyll metabolism were the most enriched KEGG pathways among the shared up-regulated DEGs (Fig. 4b, Table S8). Of the 30 genes assigned to the photosynthesis pathway, 10 encode components of the photosystem I reaction center complex, whereas 11 genes encode components of the photosystem II reaction center complex (Fig. 4c, Table 1). Additionally, one, five, and 
three genes assigned to the photosynthesis pathway are associated with the cytochrome $b 6 / f$ complex, photosynthetic electron transport, and F-type ATPases, respectively (Table 1). Twelve genes encoding light-harvesting chlorophyll proteins were assigned to the photosynthesis-antenna proteins pathway (Fig. 4c, Table 1). Moreover, 15 genes involved in chlorophyll biosynthesis were assigned to the porphyrin and chlorophyll metabolism pathway (Fig. 4c, Table 1). Other significantly enriched pathways among the up-regulated DEGs were glyoxylate and dicarboxylate metabolism, carbon fixation in photosynthetic organisms, and carbon metabolism (Fig. 4b, Table S8). These findings imply that fusion of SRDX to OsPIL11 and OsPIL16 promotes multiple important photosynthesis-related processes, including light absorption, electron transfer, and carbon assimilation. Additionally, many of the up-regulated DEGs were associated with ribosome assembly (Fig. 4b, Table S8), implying that protein synthesis is likely regulated by OsPIL11 and OsPIL16.

Plant hormone signal transduction was the most enriched KEGG pathway among the shared downregulated DEGs (Fig. 4d, Table S9). Of the 21 genes assigned to the plant hormone signal transduction pathway, 10 are associated with the auxin pathway, including seven auxin-responsive Aux/IAA genes, one small auxin up-regulated (SAUR) gene (OsSAUR38), one auxin-responsive gene (OsGH3-6), and one auxin influx carrier-encoding gene (OSAUX1) (Fig. 4e, Table 2). In addition to auxin, the down-regulated DEGs assigned to the plant hormone signal transduction pathway were associated with other hormones, including cytokinin, ethylene, abscisic acid, brassinosteroid, jasmonic acid, and salicylic acid (Table 2). Auxin regulates almost all aspects of plant growth and development essentially by modulating cell division and elongation (Santner and Estelle 2009). In Arabidopsis, PIFs may regulate auxin signaling during de-etiolation (Hornitschek et al. 2012; Nozue et al. 2011). Thus, OsPIL11-SRDX and OsPIL16-SRDX likely suppress rice skotomorphogenesis by promoting photosynthetic processes and repressing the auxin pathway in darkness.

Table 1. Differentially expressed genes assigned to the photosynthesis, photosynthesis-antenna proteins, and porphyrin and chlorophyll metabolism KEGG pathways 
log2FoldChange

$\begin{array}{lllll}\text { WTR } & \text { R11\#2 } & \text { R11\#3 } & \text { R16 } & \text { R16\#9 } \\ \text { VS } & \text { VS } & \text { VS } & \text { \#8 } & \text { VS } \\ \text { WTD } & \text { WTD } & \text { WTD } & \text { VS } & \text { WTD } \\ & & & \text { WTD } & \\ & & & & \end{array}$

Photosynthesis

\begin{tabular}{|c|c|c|c|c|c|c|}
\hline CAA33954 & $\begin{array}{l}\text { psaC/Photosystem I reaction } \\
\text { centre }\end{array}$ & 4.19 & 3.90 & 2.19 & 1.10 & 2.13 \\
\hline Os08g0560900 & $\begin{array}{l}\text { PsaD/Photosystem I reaction } \\
\text { centre }\end{array}$ & 4.14 & 3.24 & 2.42 & 2.44 & 3.10 \\
\hline Os07g0435300 & $\begin{array}{l}\text { PsaE/Photosystem I reaction } \\
\text { centre }\end{array}$ & 4.08 & 3.89 & 2.33 & 2.53 & 2.64 \\
\hline Os03g0778100 & $\begin{array}{l}\text { PsaF/Photosystem I reaction } \\
\text { centre }\end{array}$ & 4.30 & 3.94 & 2.69 & 2.80 & 3.07 \\
\hline Os09g0481200 & $\begin{array}{l}\text { PsaG/Photosystem I reaction } \\
\text { centre }\end{array}$ & 5.80 & 5.40 & 3.54 & 3.80 & 4.45 \\
\hline Os05g0560000 & $\begin{array}{l}\text { PsaH/Photosystem I reaction } \\
\text { centre }\end{array}$ & 4.06 & 3.65 & 2.61 & 3.10 & 3.33 \\
\hline Os12g0420400 & $\begin{array}{l}\text { PsaL/Photosystem I reaction } \\
\text { centre }\end{array}$ & 4.36 & 3.70 & 2.39 & 2.56 & 3.16 \\
\hline Os12g0189400 & $\begin{array}{l}\text { psaN/Photosystem I reaction } \\
\text { centre }\end{array}$ & 5.24 & 4.51 & 2.97 & 3.17 & 3.80 \\
\hline Os04g0414700 & $\begin{array}{l}\text { PsaO/Photosystem I reaction } \\
\text { centre }\end{array}$ & 5.91 & 4.86 & 3.74 & 3.36 & 4.23 \\
\hline Os07g0148900 & $\begin{array}{l}\text { PsaK/Photosystem I reaction } \\
\text { centre }\end{array}$ & 6.59 & 5.43 & 4.28 & 4.18 & 4.81 \\
\hline Os01g0501800 & $\begin{array}{l}\text { PsbO/Photosystem II reaction } \\
\text { centre }\end{array}$ & 3.11 & 2.93 & 0.94 & 1.09 & 1.45 \\
\hline Os07g0141400 & $\begin{array}{l}\text { PsbP/Photosystem II reaction } \\
\text { centre }\end{array}$ & 3.81 & 3.31 & 1.97 & 2.14 & 2.40 \\
\hline Os08g0347500 & $\begin{array}{l}\text { PsbP/Photosystem II reaction } \\
\text { centre }\end{array}$ & 2.37 & 2.21 & 0.98 & 1.18 & 1.28 \\
\hline Os07g0544800 & $\begin{array}{l}\text { PsbQ/Photosystem II reaction } \\
\text { centre }\end{array}$ & 3.76 & 3.18 & 1.79 & 2.06 & 2.41 \\
\hline Os07g0105600 & $\begin{array}{l}\text { PsbQ/Photosystem II reaction } \\
\text { centre }\end{array}$ & 2.67 & 2.34 & 0.86 & 1.44 & 1.15 \\
\hline Os08g0200300 & $\begin{array}{l}\text { PsbR/Photosystem II reaction } \\
\text { centre }\end{array}$ & 4.09 & 3.64 & 2.05 & 2.34 & 2.85 \\
\hline Os05g0508900 & PsbW/Photosystem II reaction & 5.14 & 5.70 & 4.13 & 4.63 & 4.70 \\
\hline
\end{tabular}


centre

\begin{tabular}{|c|c|c|c|c|c|c|}
\hline Os01g0773700 & $\begin{array}{l}\text { PsbW/Photosystem II reaction } \\
\text { centre }\end{array}$ & 4.00 & 3.62 & 2.14 & 2.31 & 2.74 \\
\hline Os08g0119800 & $\begin{array}{l}\text { PsbY/Photosystem II reaction } \\
\text { centre }\end{array}$ & 5.88 & 5.83 & 4.39 & 4.46 & 4.13 \\
\hline Os03g0333400 & $\begin{array}{l}\text { Pbs27/Photosystem II reaction } \\
\text { centre }\end{array}$ & 4.41 & 4.39 & 2.30 & 2.58 & 2.80 \\
\hline Os01g0938100 & $\begin{array}{l}\text { Psb28/Photosystem II reaction } \\
\text { centre }\end{array}$ & 3.04 & 3.14 & 2.14 & 2.16 & 2.41 \\
\hline Os07g0556200 & PetC/Cytochrome b6/f complex & 3.26 & 3.02 & 0.80 & 1.14 & 1.44 \\
\hline Os06g0101600 & $\begin{array}{l}\text { PetE/Photosynthetic electron } \\
\text { transport }\end{array}$ & 4.35 & 3.87 & 2.55 & 2.88 & 3.40 \\
\hline Os08g0104600 & $\begin{array}{l}\text { PetF/Photosynthetic electron } \\
\text { transport }\end{array}$ & 3.86 & 3.67 & 1.97 & 2.22 & 2.52 \\
\hline Os03g0685000 & $\begin{array}{l}\text { PetF/Photosynthetic electron } \\
\text { transport }\end{array}$ & 2.51 & 2.71 & 1.51 & 1.68 & 1.54 \\
\hline Os03g0659200 & $\begin{array}{l}\text { PetF/Photosynthetic electron } \\
\text { transport }\end{array}$ & 1.94 & 2.39 & 1.21 & 1.31 & 1.35 \\
\hline Os07g0567400 & $\begin{array}{l}\text { PetJ/Photosynthetic electron } \\
\text { transport }\end{array}$ & 1.80 & 1.73 & 0.91 & 1.20 & 1.14 \\
\hline Os07g0513000 & gamma/F-type ATPase & 3.05 & 2.38 & 1.17 & 1.32 & 1.98 \\
\hline Os02g0750100 & delta/F-type ATPase & 3.84 & 3.50 & 2.00 & 2.07 & 2.59 \\
\hline Os03g0278900 & b/F-type ATPase & 3.42 & 3.24 & 1.50 & 1.90 & 2.12 \\
\hline
\end{tabular}

Photosynthesis - antenna proteins

\begin{tabular}{|llccccc}
\hline Os07g0577600 & $\begin{array}{l}\text { Lhca2/Light-harvesting } \\
\text { chlorophyll protein complex }\end{array}$ & 4.49 & 3.93 & 2.62 & 2.24 & 2.88 \\
\hline Os09g0439500 & $\begin{array}{l}\text { Lhca6/Light-harvesting } \\
\text { chlorophyll protein complex }\end{array}$ & 3.26 & 2.79 & 1.50 & 1.69 & 2.04 \\
\hline Os08g0435900 & $\begin{array}{l}\text { Lhca4/Light-harvesting } \\
\text { chlorophyll protein complex }\end{array}$ & 8.31 & 7.45 & 5.97 & 5.57 & 6.39 \\
\hline Os06g0320500 & $\begin{array}{l}\text { Lhca1/Light-harvesting } \\
\text { chlorophyll protein complex }\end{array}$ & 6.22 & 5.17 & 3.75 & 3.55 & 4.12 \\
\hline Os02g0197600 & $\begin{array}{l}\text { Lhca3/Light-harvesting } \\
\text { chlorophyll protein complex }\end{array}$ & 5.96 & 5.18 & 3.60 & 2.93 & 3.88 \\
\hline Os02g0764500 & $\begin{array}{l}\text { Lhca5/Light-harvesting } \\
\text { chlorophyll protein complex }\end{array}$ & 3.56 & 3.27 & 1.85 & 1.83 & 2.37 \\
\hline Os01g0720500 & $\begin{array}{l}\text { Lhcb1/Light-harvesting } \\
\text { chlorophyll protein complex }\end{array}$ & 11.82 & 12.01 & 8.54 & 8.96 & 10.13
\end{tabular}




\begin{tabular}{|c|c|c|c|c|c|c|}
\hline Os11g0242800 & $\begin{array}{l}\text { Lhcb5/Light-harvesting } \\
\text { chlorophyll protein complex }\end{array}$ & 6.19 & 5.10 & 4.08 & 3.71 & 4.39 \\
\hline Os09g0346500 & $\begin{array}{l}\text { Lhcb1/Light-harvesting } \\
\text { chlorophyll protein complex }\end{array}$ & 8.20 & 7.35 & 6.54 & 6.17 & 6.73 \\
\hline Os07g0558400 & $\begin{array}{l}\text { Lhcb4/Light-harvesting } \\
\text { chlorophyll protein complex }\end{array}$ & 6.71 & 6.10 & 4.64 & 4.50 & 5.26 \\
\hline Os07g0562700 & $\begin{array}{l}\text { Lhcb3/Light-harvesting } \\
\text { chlorophyll protein complex }\end{array}$ & 5.65 & 4.69 & 3.66 & 3.09 & 4.03 \\
\hline Os03g0592500 & $\begin{array}{l}\text { Lhcb2/Light-harvesting } \\
\text { chlorophyll protein complex }\end{array}$ & 6.83 & 6.10 & 4.98 & 4.36 & 5.47 \\
\hline \multicolumn{7}{|c|}{ Porphyrin and chlorophyll metabolism } \\
\hline Os03g0563300 & $\begin{array}{l}\text { CHLI, Magnesium Chelatase } \\
\text { OsCHLI }\end{array}$ & 2.89 & 2.67 & 2.02 & 2.20 & 2.50 \\
\hline Os10g0419600 & OsCHL, chlorophyllase-2 & 4.91 & 3.41 & 1.68 & 1.95 & 2.40 \\
\hline Os01g0279100 & $\begin{array}{l}\text { YGL8, catalytic subunit of } \\
\text { magnesium-protoporphyrin IX } \\
\text { monomethyl ester cyclase }\end{array}$ & 3.83 & 3.71 & 2.49 & 2.39 & 3.00 \\
\hline Os06g0132400 & ChIM, Magnesium Chelatase & 3.23 & 3.28 & 2.53 & 2.61 & 2.81 \\
\hline Os10g0567400 & CAO, chlorophyll a oxygenase & 3.31 & 3.36 & 1.95 & 1.33 & 2.15 \\
\hline Os03g0337600 & $\begin{array}{l}\text { UroD, uroporphyrinogen } \\
\text { decarboxylase }\end{array}$ & 2.55 & 2.89 & 1.74 & 1.93 & 1.87 \\
\hline Os01g0622300 & $\begin{array}{l}\text { HEME,uroporphyrinogen } \\
\text { decarboxylase }\end{array}$ & 1.77 & 2.00 & 1.14 & 1.55 & 1.59 \\
\hline Os02g0168800 & HemC,porphobilinogen deaminase & 2.08 & 3.19 & 2.23 & 2.48 & 2.07 \\
\hline Os02g0744900 & LYL1, Geranylgeranyl Reductase & 3.36 & 3.23 & 1.93 & 1.57 & 2.18 \\
\hline Os10g0496900 & $\begin{array}{l}\text { PORB, protochlorophyllide } \\
\text { oxidoreductase B }\end{array}$ & 2.90 & 2.75 & 1.76 & 1.70 & 2.04 \\
\hline Os02g0296800 & $\begin{array}{l}\text { PF01903: CbiX, sirohydrochlorin } \\
\text { ferrochelatase }\end{array}$ & 1.42 & 1.43 & 0.78 & 1.21 & 0.98 \\
\hline Os01g0286600 & $\begin{array}{l}\text { HemY, protoporphyrinogen } \\
\text { oxidase }\end{array}$ & 1.78 & 1.82 & 1.17 & 1.27 & 1.21 \\
\hline Os05g0349700 & OsYGL1, Chlorophyll synthase & 1.48 & 1.90 & 1.16 & 1.34 & 1.21 \\
\hline Os08g0532200 & HemL,aminotransferase & 1.86 & 1.61 & 0.93 & 1.01 & 1.10 \\
\hline Os03g0351200 & $\begin{array}{l}\text { OsDVR, DVR,Divinyl Reductase } \\
\text { gene }\end{array}$ & 2.51 & 2.29 & 1.23 & 1.31 & 1.47 \\
\hline
\end{tabular}

All genes were detected as differentially expressed based on an adjusted $P<0.05$. WTR, wild-type seedlings grown under red light; WTD, wild-type seedlings grown in darkness; R11\#2 and R11\#3, dark- 
grown OsPIL11-SRDX lines \#2 and \#3, respectively; R16\#8 and R16\#9, dark-grown OsPIL 16SRDX lines \#8 and \#9, respectively.

Table 2. Differentially expressed genes assigned to the plant hormone signal transduction KEGG pathway 
Locus_ID

Gene Symbol

log2FoldChange

WTR R11\#2 vs R11\#3 R16 \#8 R16\#9

vs WTND vs WTD vs WTD vs WTD

WTD

Auxin signal pathway

Os01g0856500 OsAUX1

$-1.21 \quad-1.46$

$-1.39$

$-1.71$

$-1.52$

Os02g0805100 OsIAA9

$-3.36-5.35$

$-2.21$

$-4.56$

$-4.91$

Os03g0633500 OsIAA11

$-6.48$

$-5.09$

$-3.67$

$-3.41$

$-3.96$

Os03g0633800 OsIAA12

$-4.65 \quad-4.66$

$-2.57$

$-3.57$

$-3.68$

Os03g0742900 OsIAA13/OsIAA1

$-3.56 \quad-3.59$

$-2.49$

$-278$

$-3.43$

Os05g0143800 OsGH3-6

$-1.65 \quad-2.20$

$-1.71$

$-2.54$

$-1.62$

Os06g0166500 OsIAA20

$-3.04 \quad-5.58$

$-2.97$

$-4.57$

$-4.60$

Os09g0437400 OsSAUR38

$-4.53$

$-4.77$

$-2.83$

$-2.94$

$-2.65$

Os12g0601300 OsIAA30

$-1.36$

$-1.84$

$-1.19$

$-1.31$

$-1.41$

Os12g0601400 OsIAA3

$-3.20 \quad-3.45$

$-2.59$

$-3.17$

$-3.04$

Cytokinin signal pathway

Os11g0143300 OsRR9

$-1.28 \quad-1.36$

$-1.95$

$-1.60$

$-1.41$

Ethylene signal pathway

\begin{tabular}{llrrrrr} 
Os02g0527600 & OsCTR2 & -1.62 & -1.96 & -1.80 & -2.50 & -2.32 \\
\hline Os08g0508700 & OsEIL4 & -2.10 & -3.15 & -1.53 & -2.66 & -2.36 \\
\hline Os06g0605900 & OsFBL30 & -1.79 & -2.04 & -1.13 & -2.50 & -1.94
\end{tabular}

Abscisic acid signal pathway

\begin{tabular}{lllllll} 
Os01g0859300 & OsABI5/OREB1 & -3.55 & -3.47 & -2.33 & -2.51 & -2.64 \\
\hline Os04g0432000 & OsSAPK7 & -1.40 & -1.97 & -1.66 & -1.11 & -1.24
\end{tabular}

Brassinosteroid signal pathway

\begin{tabular}{lllllll} 
Os01g0718300 & d61/OsBRI1 & -1.09 & -1.50 & -1.04 & -1.21 & -1.15 \\
\hline Os09g0459450 & BKI1 & -0.72 & -1.43 & -0.98 & -1.74 & -1.23
\end{tabular}

Jasmonic acid signal pathway

Os08g0428400 OsJAZ3/OsTIFY6a

$-1.30 \quad-2.02$

$-1.52$

$-1.95$

$-1.55$

Salicylic acid signal pathway 


\begin{tabular}{lllllll} 
Os12g0152900 & OsbZIP83 & -3.35 & -2.83 & -2.13 & -3.15 & -1.95 \\
\hline Os07g0125500 & $\begin{array}{l}\text { Cysteine-rich secretory } \\
\text { protein family }\end{array}$ & -9.89 & -5.87 & -3.67 & -3.83 & -4.54
\end{tabular}

All genes were detected as differentially expressed based on an adjusted $P<0.05$. WTR, wild-type seedlings grown under red light; WTD, wild-type seedlings grown in darkness; R11\#2 and R11\#3, darkgrown OsPIL11-SRDX lines \#2 and \#3, respectively; R16\#8 and R16\#9, dark-grown OsPIL 16-SRDX lines \#8 and \#9, respectively.

We further confirmed the expression of genes related to photosynthesis and auxin signaling in the darkgrown OsPIL 16-SRDX and OsPIL 11-SRDX seedlings by qRT-PCR. Among 57 shared up-regulated DEGs, 36 genes related to photosynthesis, photosynthesis-antenna proteins, and porphyrin and chlorophyll metabolism pathways were analyzed in a qRT-PCR assay using rice ACTIN gene as the reference gene. The data indicated that the expression levels of all of these genes were up-regulated in the R-grown WT seedlings and in the dark-grown OSPIL 16-SRDX and OSPIL 11-SRDX seedlings (Fig. 5a, b, and c). Of the shared down-regulated DEGs assigned to the plant hormone signal transduction pathway (Table 2), eight genes related to the auxin signaling pathway were analyzed by qRT-PCR, which revealed that the expression of all eight genes was down-regulated in the R-grown WT seedlings and in the dark-grown OSPIL 16-SRDX and OSPIL 11-SRDX seedlings (Fig. 5d). Meantime, the same results were obtained in the qRT-PCR assay using eEF-1a as the reference gene (Fig. S3). The qRT-PCR results were consistent with the RNA-seq data. These findings suggest that OsPIL11-SRDX and OsPIL16-SRDX induce the expression of photosynthesis-related genes and repress the expression of genes responsive to the auxin pathway in seedlings grown in darkness.

To further assess how the shared DEGs are regulated by OsPIL11 and OsPIL16, we produced transgenic rice lines overexpressing OSPIL 11. Two independent homozygous $\mathrm{T}_{4}$ lines (\#4 and \#26) were selected to functionally characterize OSPIL11 in rice because of their high OSPIL11 expression levels (Fig. 6a). Previously reported OSPIL 16-OX lines were also used in this study (He et al. 2016). A comparison of the OsPIL11-OX, OsPIL16-OX, and WT seedlings grown in darkness for 9 days revealed that the mesocotyls of the OSPIL 17-OX and OSPIL 16-OX seedlings were significantly longer than the WT mesocotyl (Fig. 6b and $\mathrm{c}$ ), which is consistent with the skotomorphogenic phenotypes of some of the indica rice varieties (Fig. 1b). Unexpectedly, the coleoptiles of the OSPIL11-OX and OSPIL 16-OX lines were significantly shorter than the WT coleoptile (Fig. $6 \mathrm{~b}$ and c). We speculate that the limited seed reserves were mainly used by the elongating mesocotyls in the OsPIL 11-OX and OSPIL16-OX lines. These results suggest that OsPIL11 and OsPIL16 are involved in promoting rice skotomorphogenesis. We further analyzed the expression patterns of DEGs related to photosynthetic processes and the auxin pathway in OsPIL 16OX and OSPIL 11-OX seedlings grown in darkness. The expression of genes assigned to the photosynthesis, photosynthesis-antenna proteins, and porphyrin and chlorophyll metabolism KEGG pathways was significantly repressed in the OsPIL 11-OX and OsPIL 16-OX lines (Fig. 6d-f). Meantime, the same results were obtained in the qRT-PCR assay using eEF-1 $a$ as a reference gene (Fig. S4). These observations imply that OsPIL11 and OsPIL16 repress the expression of genes related to several 
important photosynthesis-related processes. The opposite effects of overexpression vs expressing SRDX fusion proteins of OsPIL11 and OsPIL16 suggest that OsPIL11 and OsPIL16 primarily function as transcriptional activators, in regards to promoting skotomorphogenesis and repressing the expression of photosynthesis-related genes.

Unexpectedly, the expression levels of genes related to auxin signaling were also down-regulated in the OSPIL 11-OX and OSPIL16-OX lines, which was consistent with the expression patterns of these genes in the OSPIL 11-SRDX and OSPIL 16-SRDX lines (Fig. 6g). These results indicate that OsPIL11 and OsPIL16 help maintain rice skotomorphogenesis by repressing the expression of genes involved in photosynthetic activities. However, whether and how the genes related to the auxin signaling pathway are regulated by OsPIL11 and OsPIL16 during rice skotomorphogenesis remains to be elucidated.

\section{Discussion}

\subsection{Fusion of the SRDX domain suppresses the functions of OsPIL11 and OsPIL16 in dark-grown rice seedlings}

In the present study, OSPIL 11-OX and OSPIL 16-OX seedlings grown in darkness had substantially elongated mesocotyls and shortened coleoptiles (Fig. 6). Mesocotyl elongation is one of the skotomorphogenic characteristics of some of the analyzed indica rice varieties (Fig. 1). Thus, ecotopic expression of OsPIL11 and OsPIL16 causes seedlings an exaggeratedly skotomorphogenic phenotypes. However, OsPIL 11-SRDX and OSPIL 16-SRDX seedlings had typical photomorphogenic phenotypes, resembling WT seedlings grown under light (Fig. 2). These observations suggest that the addition of the SRDX domain repressed the functions of OsPIL11 and OsPIL16 related to the maintenance of rice skotomorphogenesis. Consistent with these phenotypes, OsPIL11 and OsPIL16 with and without the SRDX motif had the opposite effects on gene expression patterns. For example, the expression of photosynthesis-related DEGs was repressed in the OsPIL 11-OX and OSPIL 16-OX lines, but was upregulated in the OSPIL 11-SRDX and OSPIL16-SRDX lines (Figs. 4, 5, and 6). It has been reported that that that fusion of SRDX motif to transcriptional activators converts them into dominant repressors, exhibiting phenotypic changes similar to those of plants with the corresponding loss-of-function alleles even in the presence of the original activator domain (Hiratsu et al. 2003). Based on the opposite effects of overexpression vs expressing SRDX fusion proteins for OsPIL11 and OsPIL16, we speculate that OsPIL11 and OsPIL16 function primarily as transcriptional activators, at least in regards to promoting skotomorphogenesis and repressing the expression of photosynthesis-related genes.

Notably, SRDX dominant repressors may suppress the expression of their target genes as well as the target genes of other members of the respective gene families (Hiratsu et al. 2003; Mitsuda et al. 2007). On the basis of the similarities in the photomorphogenic phenotypes and gene expression profiles of the OsPIL 11-SRDX and OSPIL 16-SRDX lines grown in darkness (Figs. 2 and 4), we assumed that the phenotypes of OSPIL11-SRDX and OSPIL 16-SRDX rice lines are probably the result of the combined effects of all or multiple OsPILs. In Arabidopsis, PIF1, PIF3, PIF4, and PIF5 function in a highly redundant 
manner to control skotomorphogenic development. For example, in contrast to the single, double, and triple pif mutants, the pifQ quadruple mutant has constitutively photomorphogenic phenotypes in darkness (Leivar et al. 2008) (Kim et al. 2011; Leivar et al. 2012; Leivar et al. 2009; Lorrain et al. 2009; Shin et al. 2009; Stephenson et al. 2009). In this context, our findings suggest that the fusion of the SRDX domain to OsPIL11 and OsPIL16 can overcome the functional redundancy of OsPIF family members.

\subsection{OsPIL11 and OsPIL16 repress the expression of photosynthetic genes}

In the present study, the expression levels of a set of genes associated with multiple steps of the photosynthetic process, including light absorption, electron transfer, and carbon assimilation, were upregulated in dark-grown OSPIL11-SRDX and OSPIL 16-SRDX lines, consistent with the corresponding expression levels in the R-grown WT seedlings, but were down-regulated in dark-grown OSPIL 11-OX and OsPIL 16-OX lines (Table 1; Fig. 5). Thus, OsPIL11 and OsPIL16 appear to repress the expression of photosynthesis-related genes in darkness. Similar results were obtained for Arabidopsis. The expression levels of most chlorophyll biosynthesis-related genes and light harvesting-related genes are up-regulated in dark-grown pifQ plants, similar to the expression levels in R-grown WT controls (Shin et al. 2009). Moreover, PIF1 and PIF3 alone repress chlorophyll biosynthesis and photosynthesis in etiolated seedlings (Shin et al. 2009). Several reports revealed the molecular mechanism by which PIFs regulate the photosynthesis genes. A previous study confirmed that the REDUCED POTASSIUM DEPENDENCY3/HISTONE DEACETYLASE1-type histone deacetylase HDA15 directly interacts with PIF3 and targets the promoters of genes involved in chlorophyll biosynthesis and photosynthesis in etiolated Arabidopsis seedlings (Liu et al. 2013). Additionally, FAR-RED ELONGATED HYPOCOTYL3 (FHY3) and FAR-RED IMPAIRED RESPONSE1 (FAR1) interact with PIF1 to regulate chlorophyll biosynthesis by modulating HEMB1 during the de-etiolation of Arabidopsis (Huq et al. 2004; Tang et al. 2012). In Arabidopsis, 24 of 103 photosynthesis-related genes are directly targeted by PIF1, PIF3, PIF4, and PIF5, but most photosynthesis-related genes are indirectly regulated by PIFs. REPRESSOR OF PHOTOSYNTHETIC GENES1 directly acts downstream of PIF1 in the endodermis to repress photosynthetic genes and regulate plastid development (Kim et al. 2016). In rice, OsPIL14 directly bound to the FLUORESCENT1 (FLU1) promoter and activated its expression to regulate chlorophyll biosynthesis in rice (Li et al. 2019). However, in the present study, the expression of FLU1 gene was up-regulated in dark-grown OSPIL 11-SRDX and OSPIL 16-SRDX lines and in the R-grown WT seedlings but were downregulated in dark-grown OSPIL 11-OX and OSPIL 16-OX lines based on the data of RNA-seq (Fig. S5). These results suggest that OsPIL11 and OsPIL16 have the opposite effects on regulating FLU1 expression compared with OsPIL14. Thus, how OsPIL11 and OsPIL16 regulate the expression photosynthesis genes is waiting for being elucidated.

\subsection{OsPIL11 and OsPIL16 represses the expression of auxin signaling pathway genes}

The down-regulated genes in the dark-grown OsPIL 11-SRDX and OSPIL 16-SRDX lines and in the R-grown WT seedlings included 10 genes associated with auxin signaling (Table 2, Figs. 5 and 6g), implying that the auxin pathway may influence rice photomorphogenesis. Several PIFs directly induce the expression of 
auxin biosynthesis-related genes, including YUCCA8 and YUCCA9, in response to shade (Hornitschek et al. 2012; Li et al. 2012a). Additionally, PIFs also regulate auxin signaling by inducing the expression of several $A U X / I A A$ and SAUR genes during de-etiolation, diurnal growth, and an exposure to shade or high temperatures (Franklin et al. 2011; Hornitschek et al. 2012; Nozue et al. 2011). In this study, the expression levels of eight DEGs associated with auxin signaling were down-regulated in the dark-grown OsPIL 11-SRDX and OSPIL 16-SRDX lines as well as in the OsPIL11-OX and OSPIL 16-OX lines (Figs. 5 and 6). It has been reported that when a transcription factor encodes a repressor, the phenotype caused by fusion of SRDX motif is similar to that of plants that overexpress the repressor but different from those with the corresponding loss-of-function alleles (Matsui et al. 2008). In this context, we speculate that OsPIL11 and OsPIL16 probably function as transcriptional repressor in regards to repressing the expression of genes related to auxin signal pathway. A potential relationship between auxin signals and skotomorphogenesis has not been established yet. However, published reports on Arabidopsis suggest that PIFs have a nonlinear relationship with auxin signaling that likely involves feedback regulatory mechanisms (Leivar and Monte 2014). As mentioned earlier, PIFs induce auxin synthesis and responses, implying they function upstream of auxin signaling. However, Chapman et al. (Chapman et al. 2012) suggested that auxin might promote growth partly through PIF-dependent pathways, implying auxin functions upstream of PIFs. Because auxin regulates almost every aspect of plant growth and development by modulating cell division and elongation (Santner and Estelle 2009), we speculate that the auxin signaling pathway is important for both skotomorphogenic and photomorphogenic development.

The KEGG pathway enrichment analysis indicated that the down-regulated DEGs were associated with hormones other than auxin (Table 2). Brassinosteroid is reportedly a skotomorphogenesis-promoting hormone that induces hypocotyl elongation in darkness and negatively regulates photomorphogenesis (Leivar and Monte 2014). In rice, D61/BRI1 is a putative brassinosteroid receptor kinase involved in brassinosteroid signaling. Moreover, the loss-of-function mutant $d 61$ exhibits defective skotomorphogenesis, implying D61/BRI1 is a positive regulator of skotomorphogenesis (Yamamuro et al. 2000). In accordance with this observation, D61 expression was significantly repressed in the dark-grown OSPIL 11-SRDX and OSPIL 16-SRDX seedlings, which had photomorphogenic phenotypes in this study (Table S2). However, whether and how other plant hormone pathways (e.g., cytokinin, ethylene, abscisic acid, jasmonic acid, and salicylic acid pathways) affect skotomorphogenesis in rice remain to be determined. Although the relationship between PIFs and plant hormones has been reported for Arabidopsis (Jeong and Choi 2013; Leivar and Monte 2014), there is relatively little information regarding this relationship in rice.

The observed phenotypes of the OSPIL 16-SRDX and OSPIL 11-SRDX seedlings were likely influenced by other rice PIF family members in addition to OsPIL11 and OsPIL16. Therefore, future studies should investigate the effects of various combinations of loss-of-function mutations to rice PIL genes on the phenotypes of plants grown in darkness. In addition, although we demonstrate that OsPIL11-SRDX and OsPIL16-SRDX suppress rice skotomorphogenesis and promotes the expression of photosynthesisrelated genes in darkness in this study, the connection between photosynthesis-related gene expression and the morphogenesis characters is waiting for being established. Clealry, mis-expression of the 
photosynthesis-related genes in the SRDX and over-expression lines of OsPIL 11 and OsPIL 16 is unlikely to account for the changes in growth and development related to photomorphogenesis and skotomorphogenesis that we observed in this study. The direct seeding of rice is increasingly being used for rice production worldwide. Therefore, elucidating the molecular mechanism underlying rice skotomorphogenic development is warranted. The genes responsible for maintaining rice skotomorphogenesis and the genes directly targeted by rice PIFs should be thoroughly investigated in future experiments.

\section{Declarations}

Funding: This work was partly supported by grants from National Natural Science Foundation of China (32070216 and 31700251).

Conflicts of interest/Competing interests (include appropriate disclosures): The authors declare that they have no conflict of interest.

Availability of data and material (data transparency): Not applicable

Code availability (software application or custom code): Not applicable.

Authors' contributions: Xianzhi Xie and Hui Zhang conceived and designed the research. Yaping Li, Fang Zhang, Chongke Zheng, Jinjun Zhou, Xiangxue Meng, Shulin Niu, and Fan Chen performed the experiments and analyzed the data. Yaping Li, Fang Zhang, and Xianzhi Xie wrote the manuscript.

Additional declarations for articles in life science journals that report the results of studies involving humans and/or animals: Not applicable

Consent for publication (include appropriate statements): All authors read this paper and confirm the context.

\section{Acknowledgment}

We thank Liwen Bianji, Edanz Editing China (www.liwenbianji.cn/ac) for editing the English text of a draft of this manuscript.

\section{References}

1. Castillon A, Shen H, Huq E (2007) Phytochrome Interacting Factors: central players in phytochromemediated light signaling networks. Trends Plant Sci 12:514-521 .

https://doi.org/10.1016/j.tplants.2007.10.001

2. Chapman EJ, Greenham K, Castillejo C, Sartor R, Bialy A, Sun TP, Estelle M (2012) Hypocotyl transcriptome reveals auxin regulation of growth-promoting genes through GA-dependent and independent pathways. PloS one 7:e36210. https://doi.org/10.1371/journal.pone.0036210 
3. Chen D, Xu G, Tang W, Jing Y, Ji Q, Fei Z, Lin R (2013) Antagonistic basic helix-loop-helix/bZIP transcription factors form transcriptional modules that integrate light and reactive oxygen species signaling in Arabidopsis. Plant cell 25:1657-1673. https://doi.org/10.1105/tpc.112.104869

4. Franklin KA, Lee SH, Patel D, Kumar SV, Spartz AK, Gu C, Ye S, Yu P, Breen G, Cohen JD, Wigge PA, Gray WM (2011) Phytochrome-interacting factor 4 (PIF4) regulates auxin biosynthesis at high temperature. Proc Natl Acad Sci U S A 108:20231-20235. https://doi.org/10.1073/pnas.1110682108

5. He Y, Li Y, Cui L, Xie L, Zheng C, Zhou G, Zhou J, Xie X (2016) Phytochrome B Negatively Affects Cold Tolerance by Regulating OsDREB1 Gene Expression through Phytochrome Interacting Factor-Like Protein OsPIL16 in Rice. Front Plant Sci 7:1963. https://doi.org/10.3389/fpls.2016.01963

6. Hiratsu K, Ohta M, Matsui K, Ohme-Takagi M (2002) The SUPERMAN protein is an active repressor whose carboxy-terminal repression domain is required for the development of normal flowers. FEBS Lett. 514:351-4. https://doi.org/ 10.1016/s0014-5793(02)02435-3

7. Hiratsu K, Matsui K, Koyama T, Ohme-Takagi M (2003) Dominant repression of target genes by chimeric repressors that include the EAR motif, a repression domain, in Arabidopsis. Plant J 34:733739. https://doi.org/10.1046/j.1365-313x.2003.01759.x

8. Hornitschek P, Kohnen MV, Lorrain S, Rougemont J, Ljung K, López-Vidriero I, Franco-Zorrilla JM, Solano R, Trevisan M, Pradervand S, Xenarios I, Fankhauser C (2012) Phytochrome interacting factors 4 and 5 control seedling growth in changing light conditions by directly controlling auxin signaling. Plant J 71:699-711. https://doi.org/10.1111/j.1365-313X.2012.05033.X

9. Huq E, Al-Sady B, Hudson M, Kim C, Apel K, Quail PH (2004) PHYTOCHROME-INTERACTING FACTOR 1 is a critical bHLH regulator of chlorophyll biosynthesis. Science 305:1937-1941. https://doi.org/10.1126/science.1099728

10. Jain M, Nijhawan A, Tyagi AK, Khurana JP (2006) Validation of housekeeping genes as internal control for studying gene expression in rice by quantitative real-time PCR. Biochem Biophys Res Commun 345:646-651. https://doi.org/10.1016/j.bbrc.2006.04.140

11. Jeong J, Choi G (2013) Phytochrome-interacting factors have both shared and distinct biological roles. Mol Cells 35:371-380. https://doi.org/10.1007/s10059-013-0135-5

12. Josse EM, Halliday KJ (2008) Skotomorphogenesis: the dark side of light signalling. Curr Biol 18:R1144-1146. https://doi.org/10.1016/j.cub.2008.10.034

13. Kim K, Jeong J, Kim J, Lee N, Kim ME, Lee S, Chang Kim S, Choi G (2016) PIF1 Regulates Plastid Development by Repressing Photosynthetic Genes in the Endodermis. Mol Plant 9:1415-1427. https://doi.org/10.1016/j.molp.2016.08.007

14. Kim K, Shin J, Lee SH, Kweon HS, Maloof JN, Choi G (2011) Phytochromes inhibit hypocotyl negative gravitropism by regulating the development of endodermal amyloplasts through phytochromeinteracting factors. Proc Natl Acad Sci U S A 108:1729-1734. https://doi.org/10.1073/pnas.1011066108

15. Langmead B, Salzberg SL (2012) Fast gapped-read alignment with Bowtie 2. Nat Methods 9:357359. https://doi.org/10.1038/nmeth.1923 
16. Lee N, Choi G (2017) Phytochrome-interacting factor from Arabidopsis to liverwort. Curr Opin Plant Biol 35:54-60. https://doi.org/10.1016/j.pbi.2016.11.004

17. Leivar P, Monte E (2014) PIFs: systems integrators in plant development. Plant cell 26:56-78. https://doi.org/10.1105/tpc.113.120857

18. Leivar $P$ et al. (2008) Multiple phytochrome-interacting bHLH transcription factors repress premature seedling photomorphogenesis in darkness. Curr Biol 18:1815-1823. https://doi.org/10.1016/j.cub.2008.10.058

19. Leivar P, Quail PH (2011) PIFs: pivotal components in a cellular signaling hub. Trends Plant Sci 16:19-28. https://doi.org/10.1016/j.tplants.2010.08.003

20. Leivar P, Tepperman JM, Cohn MM, Monte E, Al-Sady B, Erickson E, Quail PH (2012) Dynamic antagonism between phytochromes and PIF family basic helix-loop-helix factors induces selective reciprocal responses to light and shade in a rapidly responsive transcriptional network in Arabidopsis. Plant cell 24:1398-1419. https://doi.org/10.1105/tpc.112.095711

21. Leivar P, Tepperman JM, Monte E, Calderon RH, Liu TL, Quail PH (2009) Definition of early transcriptional circuitry involved in light-induced reversal of PIF-imposed repression of photomorphogenesis in young Arabidopsis seedlings. Plant cell 21:3535-3553. https://doi.org/10.1105/tpc.109.070672

22. Li B, Dewey CN (2011) RSEM: accurate transcript quantification from RNA-Seq data with or without a reference genome. BMC bioinformatics 12:323. https://doi.org/10.1186/1471-2105-12-323

23. Li L, Ljung K, Breton G, Schmitz RJ, Pruneda-Paz J, Cowing-Zitron C, Cole BJ, Ivans LJ, Pedmale UV, Jung HS, Ecker JR, Kay SA, ChoryJ (2012a) Linking photoreceptor excitation to changes in plant architecture. Genes Dev 26:785-790. https://doi.org/10.1101/gad.187849.112

24. Li L, Peng W, Liu Q, Zhou J, Liang W, Xie X (2012b) Expression Patterns of OsPIL 11, a PhytochromeInteracting Factor in Rice, and Preliminary Analysis of Its Roles in Light Signal Transduction. Rice Sci 19:263-268. https://doi.org/10.1016/S1672-6308(12)60050-X

25. Li Z, Mo W, Jia L, Xu YC, Tang W, Yang W, Guo YL, Lin R (2019) Rice FLUORESCENT1 Is Involved in the Regulation of Chlorophyll. Plant Cell Physiol 60:2307-2318. https://doi.org/10.1093/pcp/pcz129

26. Livak K J, Schmittgen T D (2001) Analysis of relative gene expression data using real-time quantitative PCR and the 2(-Delta Delta C(T)) Method. Methods 25:402-408. https://doi.org/10.1006/meth.2001.1262

27. Liu X, Chen CY, Wang KC, Luo M, Tai R, Yuan L, Zhao M, Yang S, Tian G, Cui Y, Hsieh HL, Wu K (2013) PHYTOCHROME INTERACTING FACTOR3 associates with the histone deacetylase HDA15 in repression of chlorophyll biosynthesis and photosynthesis in etiolated Arabidopsis seedlings. Plant cell 25:1258-1273. https://doi.org/10.1105/tpc.113.109710

28. Lorrain S, Trevisan M, Pradervand S, Fankhauser C (2009) Phytochrome interacting factors 4 and 5 redundantly limit seedling de-etiolation in continuous far-red light. Plant $\mathrm{J}$ 60:449-461. https://doi.org/10.1111/j.1365-313X.2009.03971.x 
29. Love MI, Huber W, Anders S (2014) Moderated estimation of fold change and dispersion for RNA-seq data with DESeq2. Genome Biol 15:550. https://doi.org/10.1186/s13059-014-0550-8

30. Matsui K, Umemura Y, Ohme-Takagi M (2008) AtMYBL2, a protein with a single MYB domain, acts as a negative regulator of anthocyanin biosynthesis in Arabidopsis. Plant $\mathrm{J}$ 55:954-967. https://doi.org/10.1111/j.1365-313X.2008.03565.x

31. Mitsuda N, Iwase A, Yamamoto H, Yoshida M, Seki M, Shinozaki K, Ohme-Takagi M (2007) NAC transcription factors, NST1 and NST3, are key regulators of the formation of secondary walls in woody tissues of Arabidopsis. Plant cell 19:270-280. https://doi.org/10.1105/tpc.106.047043

32. Murray MG, Thompson WF (1980) Rapid isolation of high molecular weight plant DNA. Nucleic Acids Res 8:4321-4325. https://doi.org/10.1093/nar/8.19.4321

33. Nakamura Y, Kato T, Yamashino T, Murakami M, Mizuno T (2007) Characterization of a set of phytochrome-interacting factor-like bHLH proteins in Oryza sativa. Biosci Biotechnol Biochem 71:1183-1191. https://doi.org/10.1271/bbb.60643

34. Nozue K, Harmer SL, Maloof JN (2011) Genomic analysis of circadian clock-, light-, and growthcorrelated genes reveals PHYTOCHROME-INTERACTING FACTOR5 as a modulator of auxin signaling in Arabidopsis. Plant Physiol 156:357-372. https://doi.org/10.1104/pp.111.172684

35. Ohta M, Matsui K, Hiratsu K, Shinshi H, Ohme-Takagi M (2001) Repression domains of class II ERF transcriptional repressors share an essential motif for active repression. Plant Cell. 13:1959-68. https://doi.org/10.1105/tpc.010127.

36. Pham VN, Kathare PK, Huq E (2018) Phytochromes and Phytochrome Interacting Factors. Plant Physiol 176:1025-1038. https://doi.org/10.1104/pp.17.01384

37. Santner A, Estelle M (2009) Recent advances and emerging trends in plant hormone signalling. Nature 459:1071-1078. https://doi.org/10.1038/nature08122

38. Shin J, Kim K, Kang H, Zulfugarov IS, Bae G, Lee CH, Lee D, Choi G (2009) Phytochromes promote seedling light responses by inhibiting four negatively-acting phytochrome-interacting factors. Proc Natl Acad Sci U S A 106:7660-7665. https://doi.org/10.1073/pnas.0812219106

39. Stephenson PG, Fankhauser C, Terry MJ (2009) PIF3 is a repressor of chloroplast development. Proc Natl Acad Sci U S A 106:7654-7659. https://doi.org/10.1073/pnas.0811684106

40. Tang W, Wang W, Chen D, Ji Q, Jing Y, Wang H, Lin R (2012) Transposase-derived proteins FHY3/FAR1 interact with PHYTOCHROME-INTERACTING FACTOR1 to regulate chlorophyll biosynthesis by modulating HEMB1 during deetiolation in Arabidopsis. Plant cell 24:1984-2000. https://doi.org/10.1105/tpc.112.097022

41. Yamamuro $C$, Ihara $Y$, Wu X, Noguchi T, Fujioka S, Takatsuto S, Ashikari M, Kitano H, Matsuoka M (2000) Loss of function of a rice brassinosteroid insensitive1 homolog prevents internode elongation and bending of the lamina joint. Plant cell 12:1591-1606. https://doi.org/10.1105/tpc.12.9.1591

42. Yu G, Wang LG, Han Y, He QY (2012) clusterProfiler: an R package for comparing biological themes among gene clusters. OMICS 16:284-287. https://doi.org/10.1089/omi.2011.0118 
43. Zhang Y, Mayba O, Pfeiffer A, Shi H, Tepperman JM, Speed TP, Quail PH (2013) A quartet of PIF bHLH factors provides a transcriptionally centered signaling hub that regulates seedling morphogenesis through differential expression-patterning of shared target genes in Arabidopsis. PLoS Genet 9:e1003244. https://doi.org/10.1371/journal.pgen.1003244

\section{Figures}

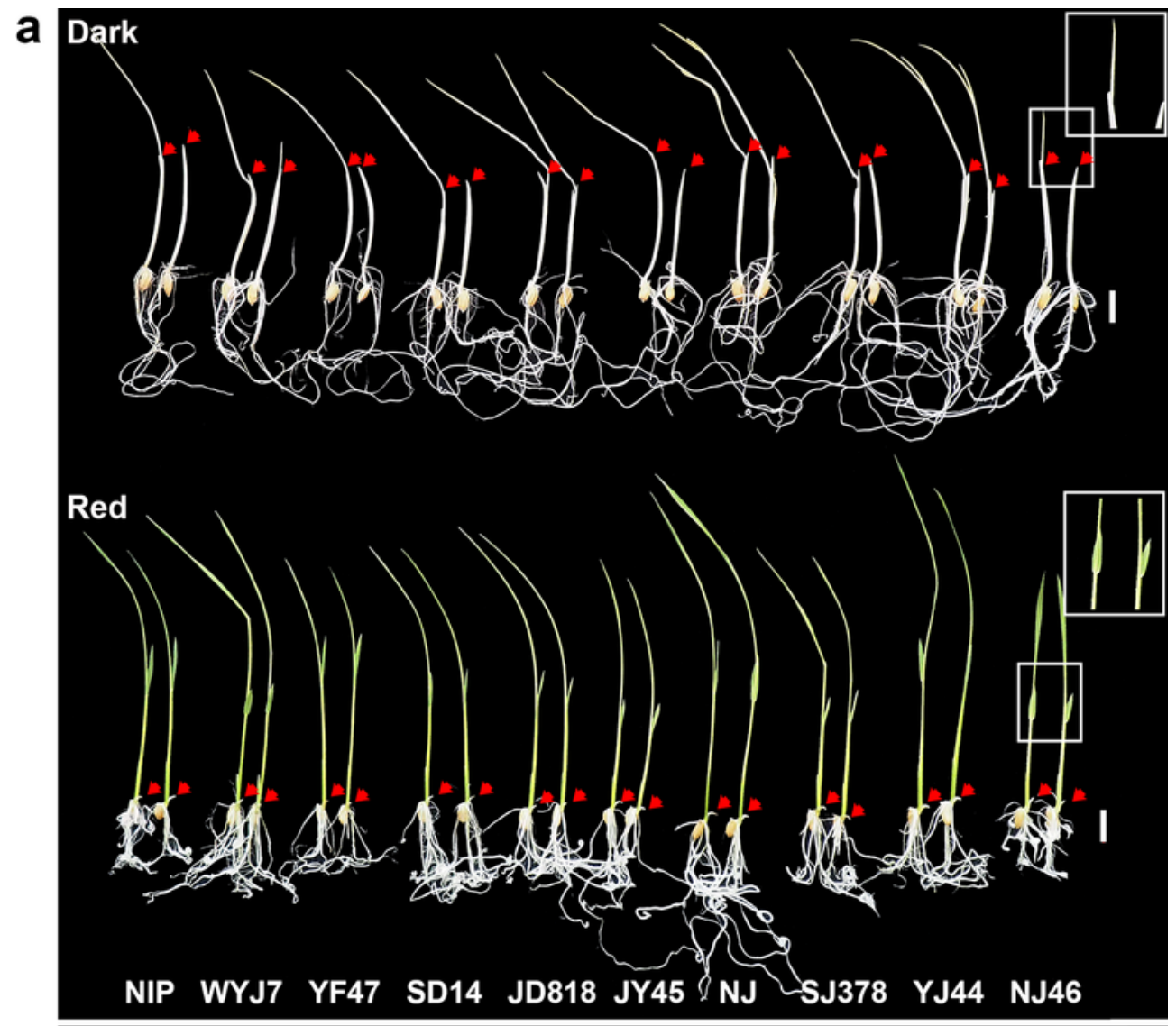

b

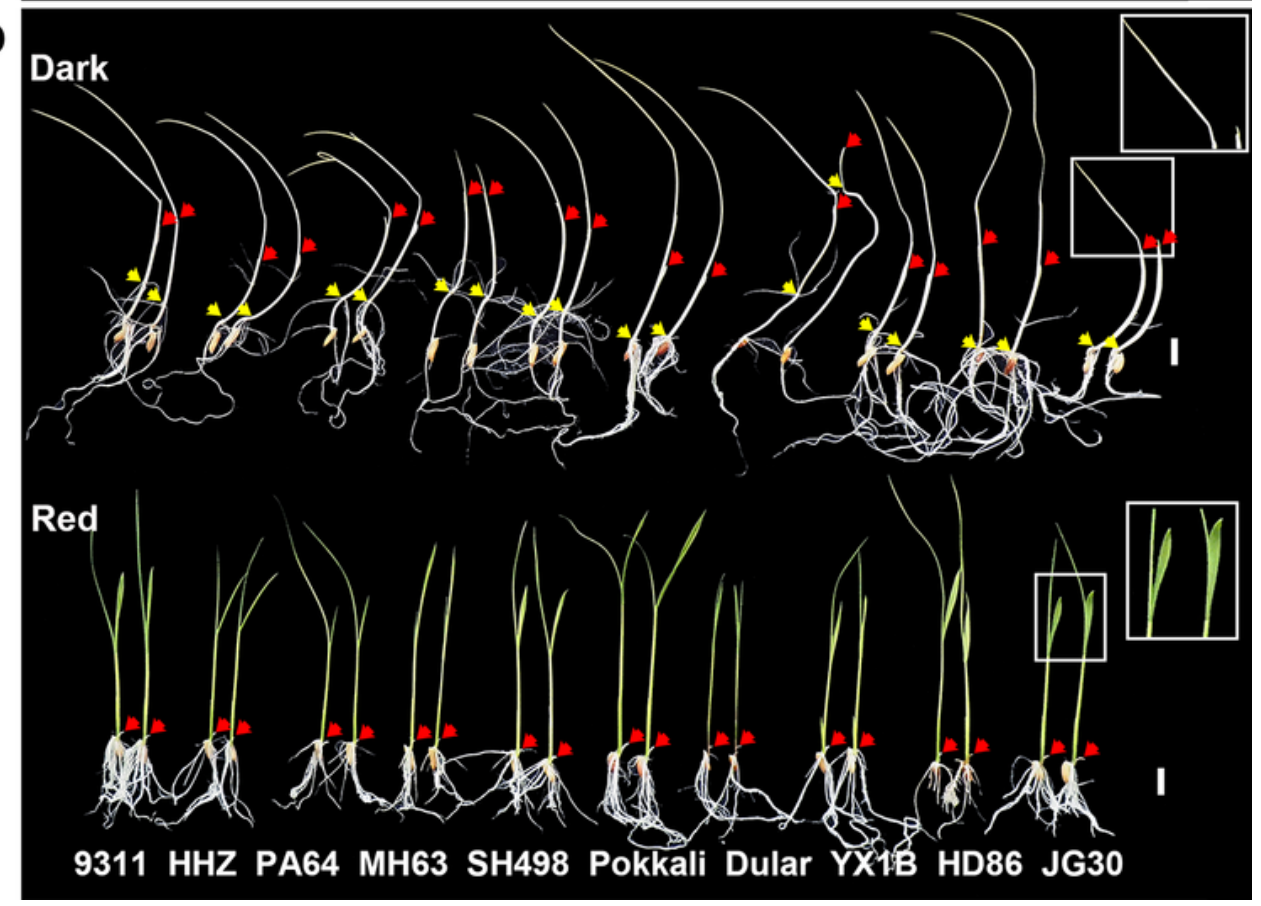


Phenotypes of etiolated rice seedlings grown under continuous light or in darkness for 9 days (a) Representative phenotypes of japonica rice varieties grown in darkness (upper panel) or under continuous red light (lower panel) for 9 days. NIP, Nipponbare; WYJ7, Wuyunjing7; YF47, Yanfeng47; SD14, Shengdao14; JD818, Jindao818; JY45, Jinyuan45; NJ, Ningjing44; SJ378, Songjing378; YJ44, Yanjing44; NJ46, Nanjing46. (b) Representative phenotypes of indica rice varieties. HHZ, Huanghuazhan; PA64, Peiai64; MH63, Minghui63; SH498, Shuhui498; YX1B, Yixiang1B; HD86, Haidao86; JG30, Jingang30. Red arrows indicate the coleoptile apex. Yellow arrows indicate the mesocotyl apex. Scale bar $=1 \mathrm{~cm}$.

a
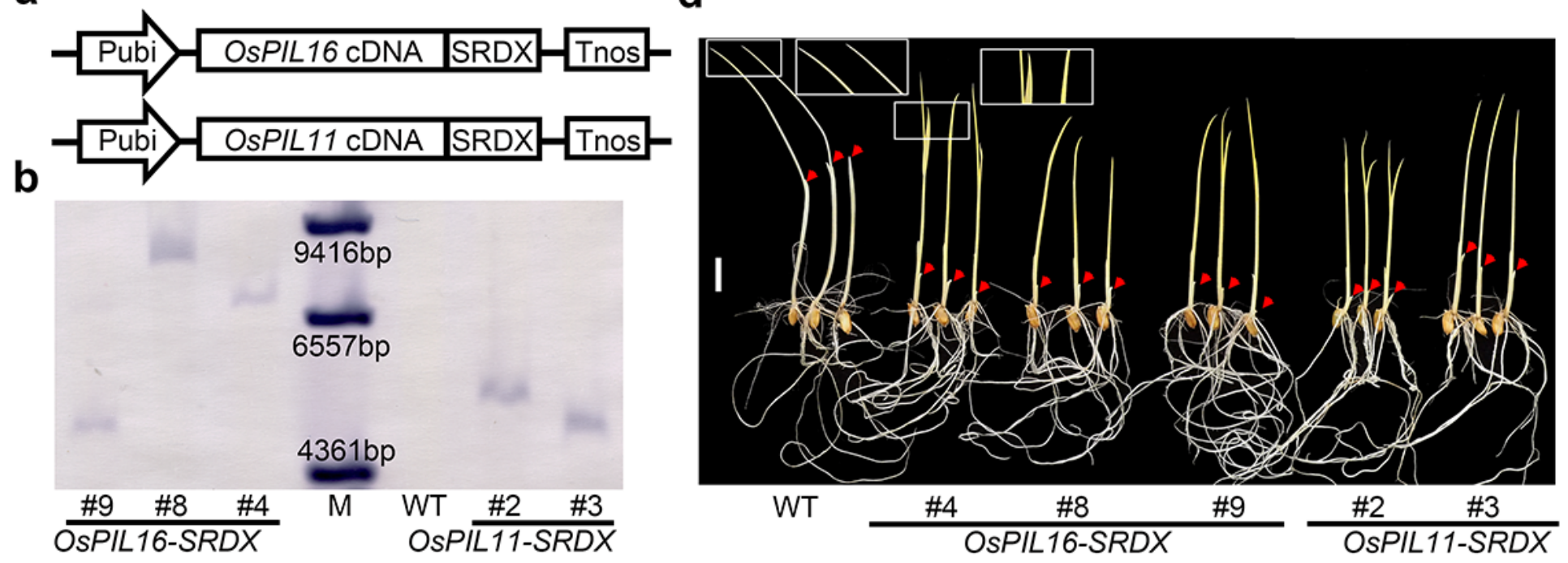

C
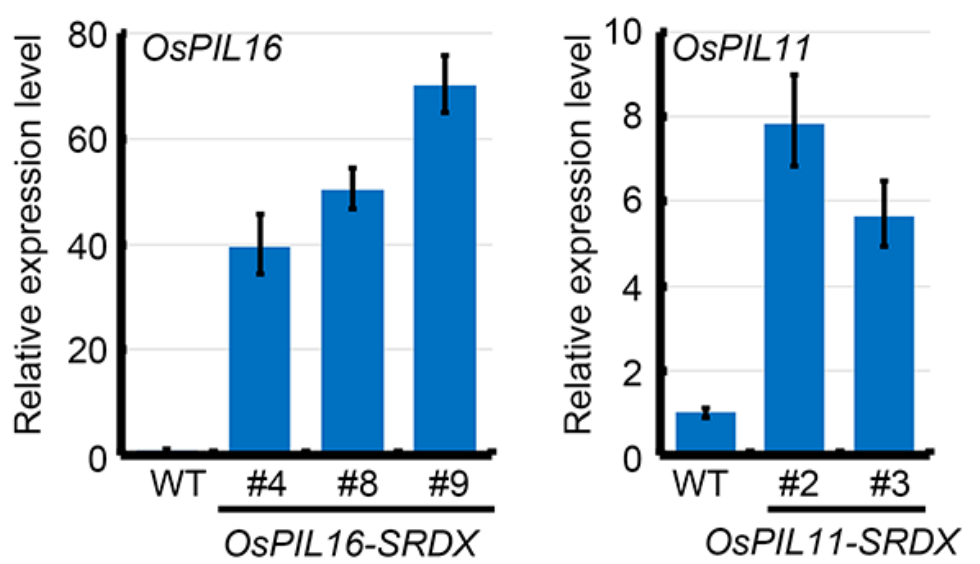

e

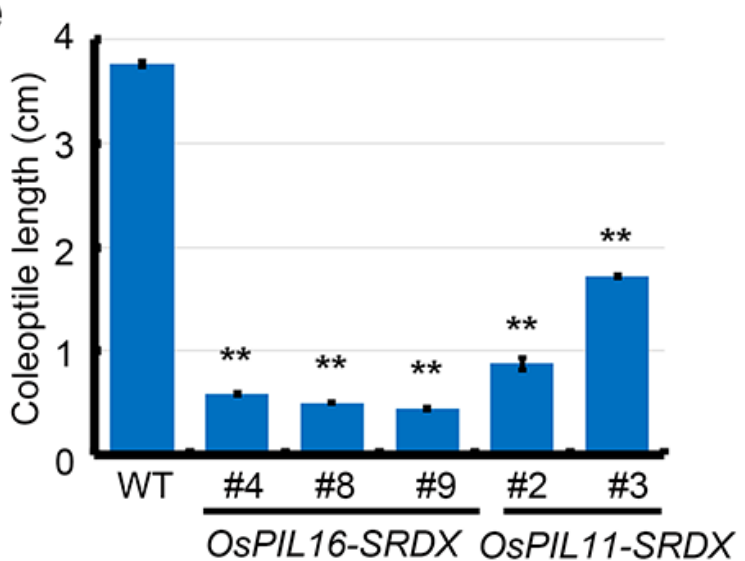

Figure 2

OsPIL16-SRDX and OsPIL11-SRDX seedlings grown in darkness had photomorphogenic phenotypes (a) Schematic diagram of the construct used to produce OsPIL16-SRDX and OsPIL11-SRDX lines. The expression of OsPIL16-SRDX was driven by the maize ubiquitin gene promoter (Pubi). (b) Southern blot analysis of three independent OsPIL16-SRDX lines (\#4, \#8, and \#9), two independent OsPIL11-SRDX lines (\#2 and \#3) and wild-type (WT) seedlings with selection marker gene, hygromycin phosphotransferase II, as probe. M, DNA molecular marker. (c) OsPIL11 transcript levels in two independent OsPIL11-SRDX lines (\#2 and \#3) and OsPIL16 transcript levels in three independent OsPIL16-SRDX lines (\#4, \#8, and \#9). (d) 
Visible phenotypes of three independent OsPIL16-SRDX lines (\#4, \#8, and \#9), two independent OsPIL11SRDX lines (\#2 and \#3) and WT seedlings grown in darkness for 9 days. Red arrows indicate the coleoptile apex. Scale bar $=1 \mathrm{~cm}$. (e) Coleoptile lengths of WT, OsPIL16-SRDX (\#4, \#8, and \#9) and OsPIL11-SRDX (\#2 and \#3) seedlings grown in darkness for 9 days. Data are presented as the mean \pm standard error for 20-30 seedlings. ** $\mathrm{P}<0.01$ compared with the WT control (Student's t-test).

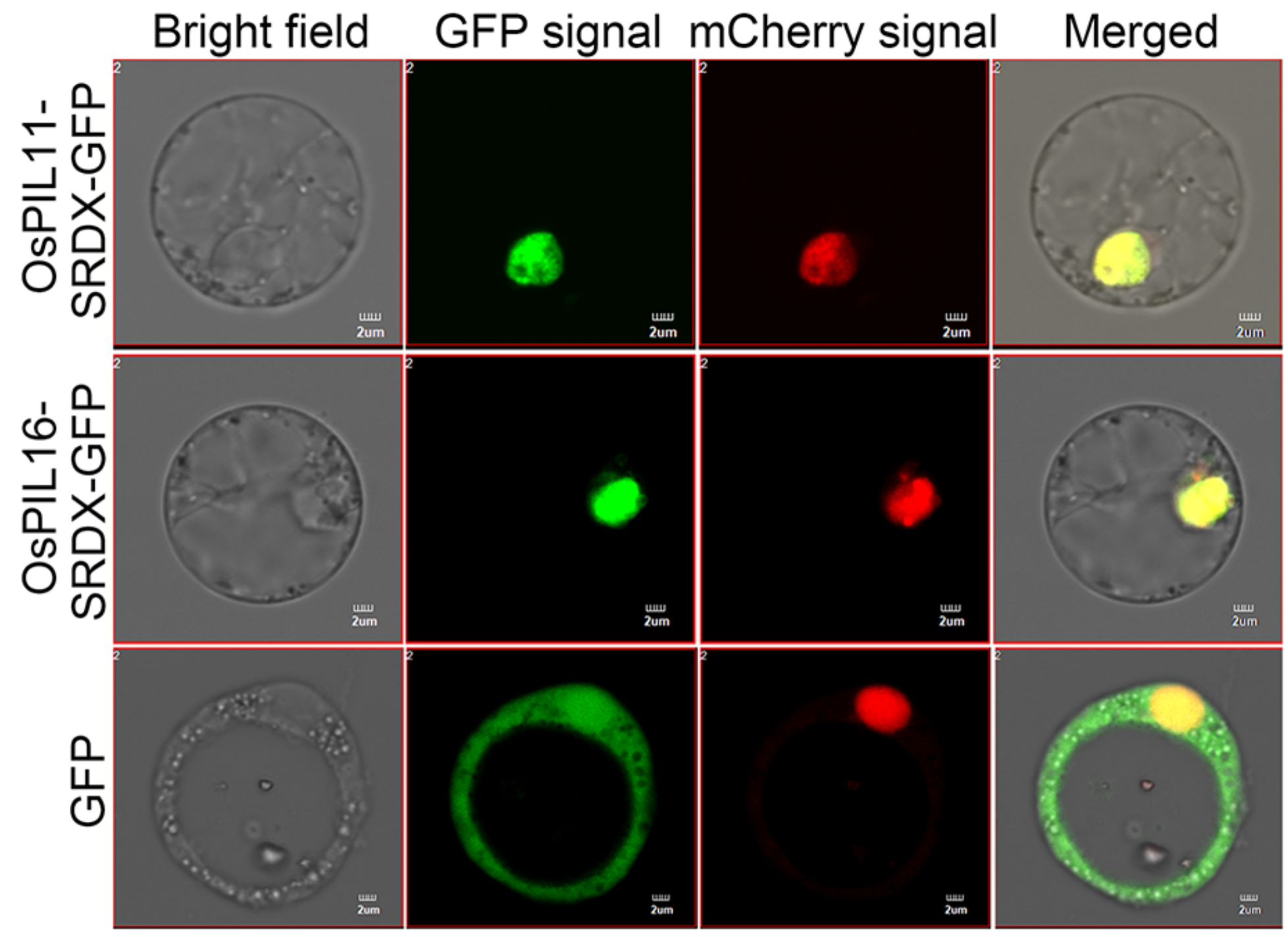

Figure 3

Nuclear localization of the OsPIL11-SRDX and OsPIL16-SRDX proteins Transient expression of OsPIL11SRDX-GFP, OsPIL16-SRDX-GFP, and GFP alone in rice protoplasts. The OsGEN1-mCherry protein was used as a nuclear marker. GFP, green fluorescent protein. 
a

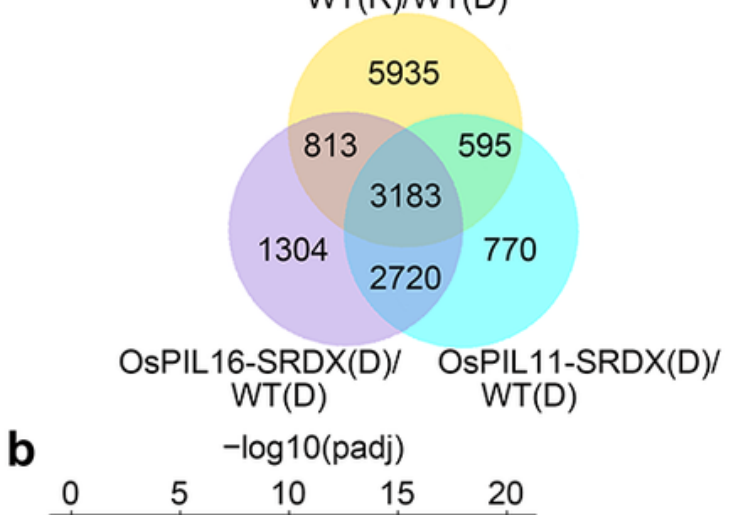

Photosynthesis

Photosynthesis - antenna proteins Porphyrin and chlorophyll metabolism Ribosome

Glyoxylate and dicarboxylate metabolism Carbon fixation in photosynthetic organisms Carbon metabolism

d

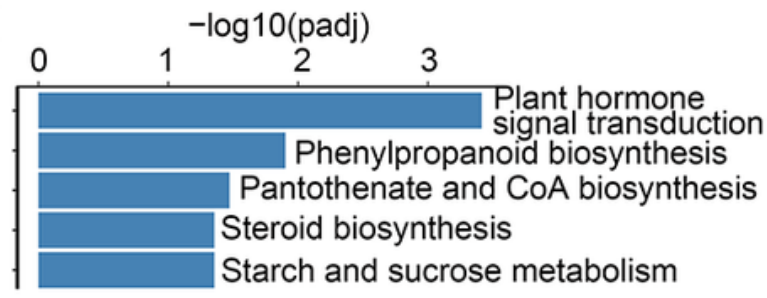

e

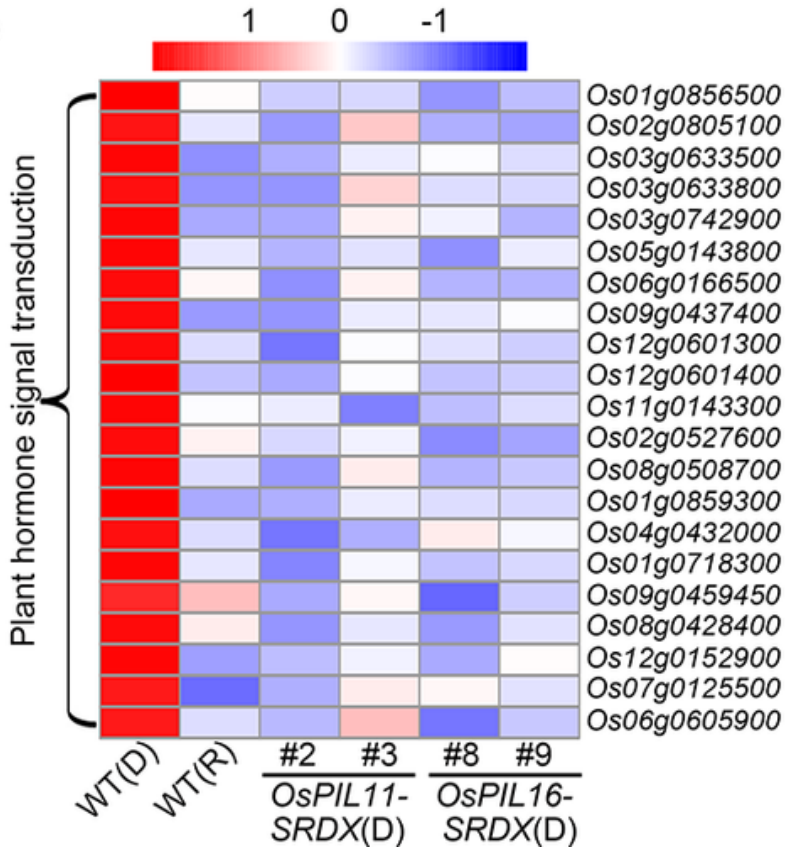

C

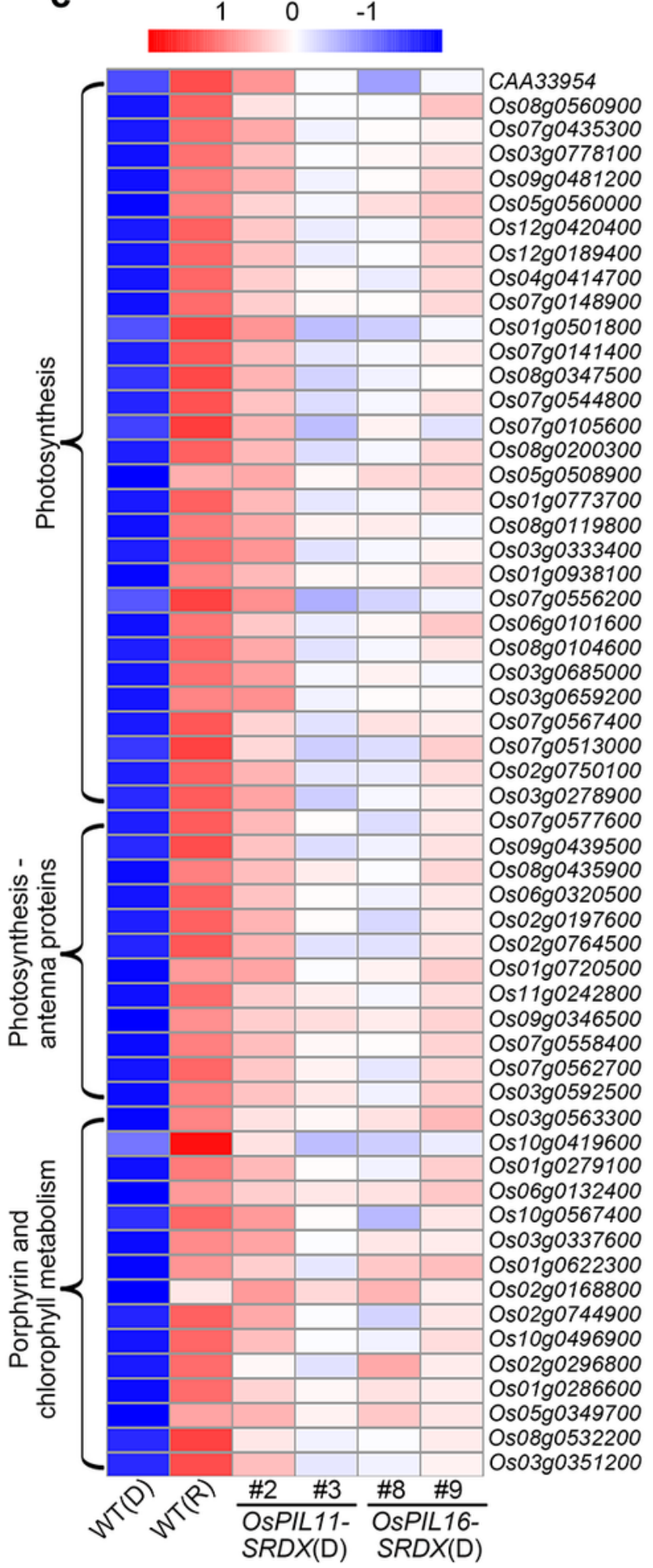

\section{Figure 4}

Dark-grown OsPIL16-SRDX and OsPIL11-SRDX seedlings express a subset of red light-regulated genes (a) Venn diagram presenting the shared differentially expressed genes (DEGs) among WT(R)/WT(D), OsPIL16-SRDX(D)/WT(D), and OsPIL11-SRDX(D)/WT(D). The DEGs were detected based on an expression level fold-change $\geq 2$ and an adjusted $P<0.05$. (b) KEGG pathway enrichment analysis of the shared up-regulated DEGs. Enriched KEGG pathways were detected based on an adjusted P < 0.05. (c) 
Heatmap of the expression level fold-changes (based on RNA-seq data) of the up-regulated DEGs assigned to the most enriched KEGG pathways. (d) KEGG pathway enrichment analysis of shared downregulated DEGs. Enriched KEGG pathways were detected based on an adjusted $P<0.05$. (e) Heatmap of the expression level fold-changes (based on RNA-seq data) of the down-regulated DEGs assigned to the most enriched KEGG pathways.
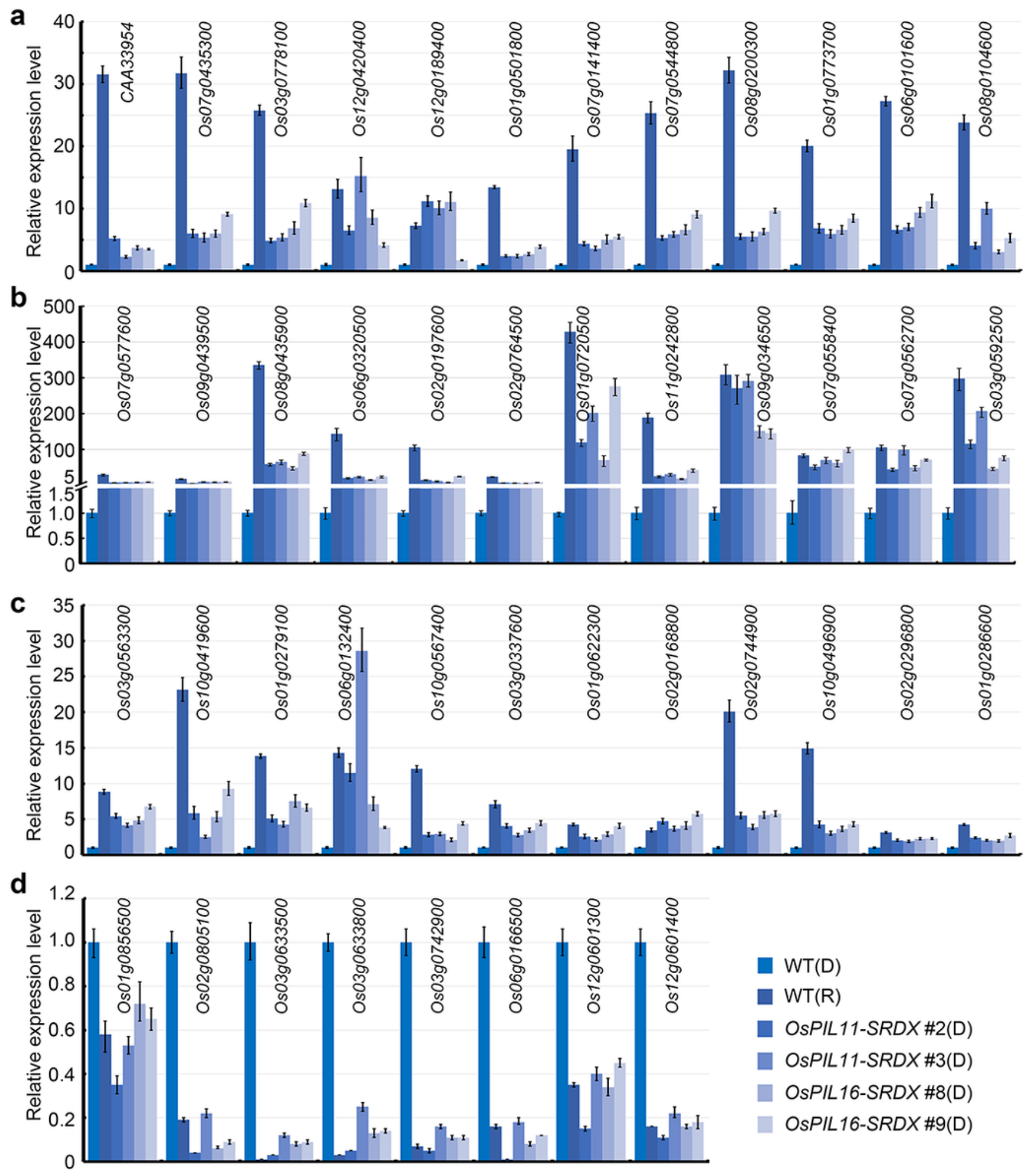

Figure 5 
Analysis of selected differentially expressed genes (DEGs) by quantitative real-time (qRT)-PCR (a-c)

Results of the qRT-PCR analysis of the up-regulated DEGs assigned to the photosynthesis (a),

photosynthesis-antenna proteins (b), and porphyrin and chlorophyll metabolism (c) KEGG pathways. (d)

Results of the qRT-PCR analysis of the down-regulated DEGs related to the auxin signaling pathway. Total RNA was isolated from wild-type (WT) seedlings grown in darkness or under red light for 6 days and from OsPIL11-SRDX and OsPIL16-SRDX seedlings grown in darkness for 6 days. Expression levels of genes related to photosynthesis and the auxin pathway were determined by qRT-PCR. The ACTIN gene was used as an internal control. Bars indicate the standard deviation in three replicates. 

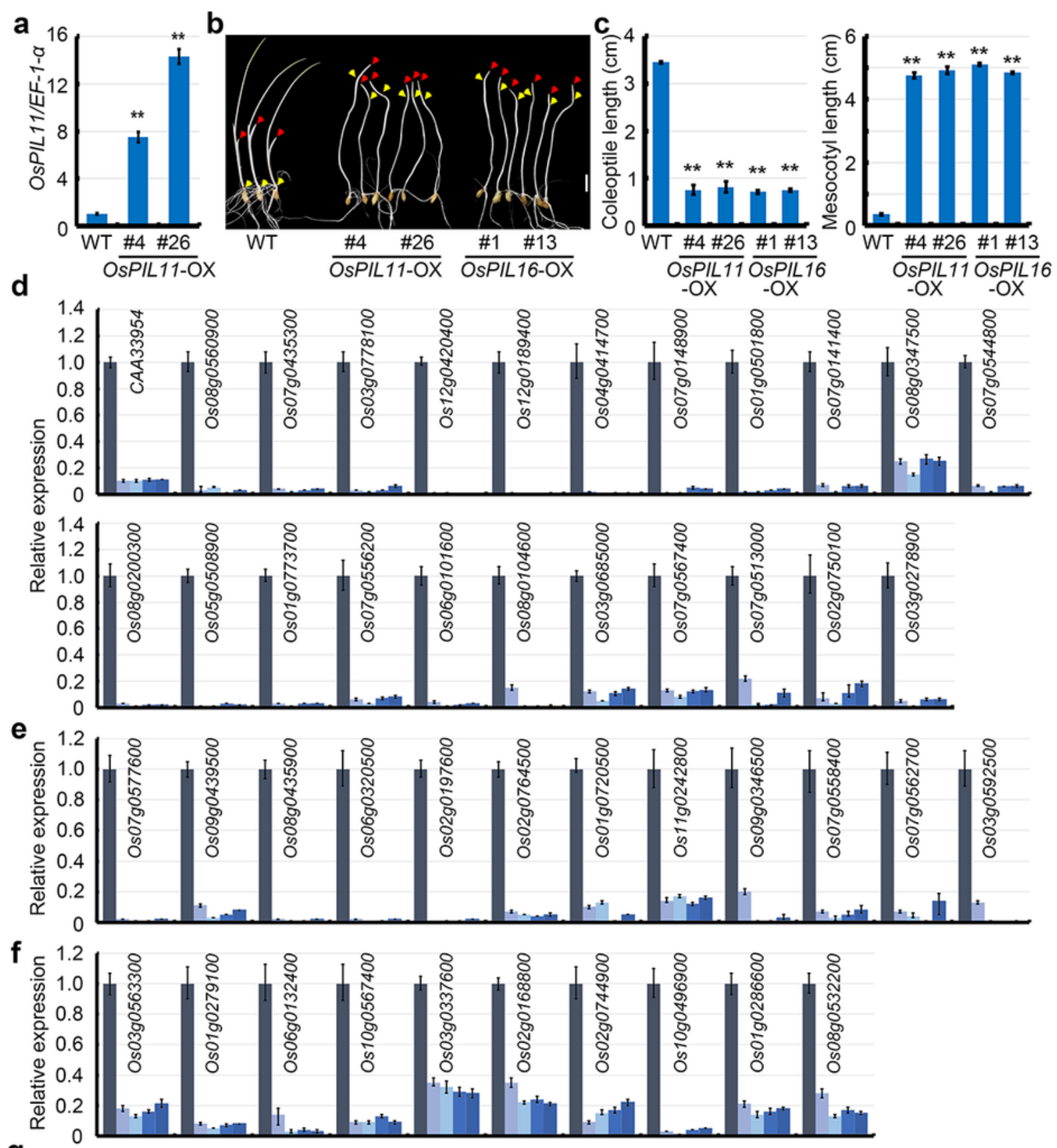

g

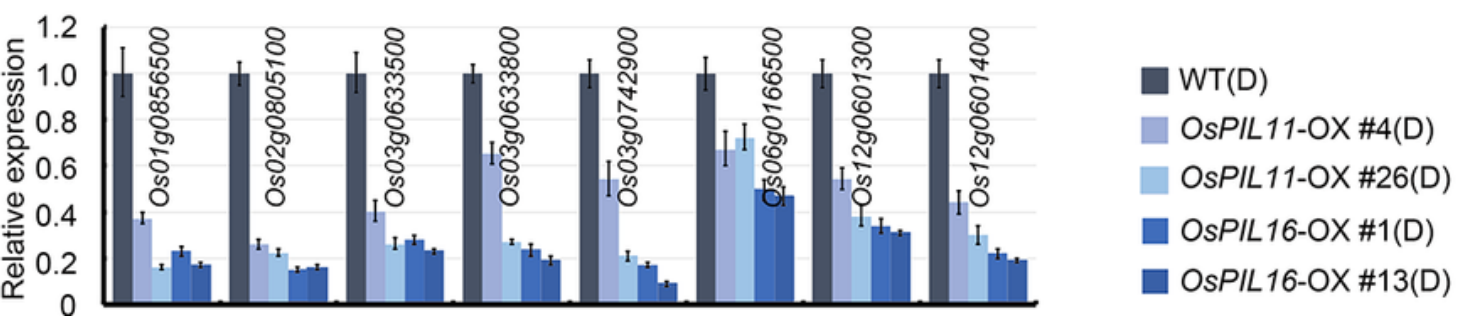

\section{Figure 6}

Skotomorphogenic phenotypes and analysis of selected differentially expressed genes (DEGs) by quantitative real-time (qRT)-PCR in dark-grown OsPIL11-OX and OsPIL16-OX seedlings (a) OsPIL11 transcript levels in two independent OsPIL11-OX lines (\#4 and \#26). (b) Visible phenotypes of two independent OsPIL11-OX lines (\#4 and \#26), two independent OsPIL16-OX lines (\#1 and \#13), and wildtype (WT) seedlings grown in darkness for 9 days. Red arrows indicate the coleoptile apex. Yellow arrows 
indicate the mesocotyl apex. Scale bar $=1 \mathrm{~cm}$. (c) Mesocotyl and coleoptile lengths of the WT, OsPIL11OX, and OsPIL16-OX seedlings grown in darkness for 9 days. Data are presented as the mean \pm standard error for 20-30 seedlings. ${ }^{* * P}<0.01$ compared with the WT control (Student's t-test). (d-f) qRT-PCR analysis of the up-regulated DEGs assigned to the photosynthesis (d), photosynthesis-antenna proteins (e), and porphyrin and chlorophyll metabolism (f) KEGG pathways. (g) qRT-PCR analysis of the downregulated DEGs related to the auxin signaling pathway. Total RNA was isolated from WT, OsPIL11-OX, and OsPIL16-OX seedlings grown in darkness for 6 days. The ACTIN gene was used as an internal control. Bars indicate the standard deviation in three replicates.

\section{Supplementary Files}

This is a list of supplementary files associated with this preprint. Click to download.

- Supplementarydata.rar 